\title{
Féeries
}

Études sur le conte merveilleux, XVII $-\mathrm{XIX}{ }^{\mathrm{e}}$ siècle

\section{Un conte vénitien magnétique. Mémoire et histoire dans L'Entrevue de Henri de Régnier}

A Magnetic Venitian Tale. Memory and History in L'Entrevue of Henri de Régnier

Jean-François Perrin

\section{OpenEdition}

Journals

Édition électronique

URL : https://journals.openedition.org/feeries/3859

DOI : 10.4000/feeries.3859

ISSN : $1957-7753$

Éditeur

UGA Éditions/Université Grenoble Alpes

Édition imprimée

ISBN : 978-2-37747-327-4

ISSN : 1766-2842

\section{Référence électronique}

Jean-François Perrin, « Un conte vénitien magnétique. Mémoire et histoire dans L'Entrevue de Henri de

Régnier », Féeries [En ligne], 17 | 2021, mis en ligne le 17 décembre 2021, consulté le 13 janvier 2023.

URL : http://journals.openedition.org/feeries/3859; DOI : https://doi.org/10.4000/feeries.3859

Ce document a été généré automatiquement le 13 janvier 2023.

Tous droits réservés 


\title{
Un conte vénitien magnétique. Mémoire et histoire dans L'Entrevue de Henri de Régnier
}

\author{
A Magnetic Venitian Tale. Memory and History in L'Entrevue of Henri de \\ Régnier
}

Jean-François Perrin

1 Henri de Régnier a rédigé L'Entrevue à Paris, alors que la première guerre mondiale venait d'éclater, l'empêchant de retourner à Venise où il avait passé l'automne précédent :

Je pris mes quartiers au palais Vendramin ai Carmini, sur la Fondamenta Foscarini, - le palais Altinengo de l'Entrevue. C'était en 1913 et ce n'est pas un fantôme du passé qui eût dû m'y apparaître en ses troubles miroirs, mais plutôt le spectre sanglant de l'avenir. En 1914 la guerre éclatait et allait, pendant plusieurs années, m'éloigner de Venise ${ }^{1}$.

Ce récit fut publié en 1919 au Mercure de France, dans un recueil intitulé Histoires incertaines. En 1929, L'Entrevue fut repris dans une anthologie regroupant ses Contes vénitiens. Dans la préface qu'il y donna, Régnier revient sur le plaisir que sa rédaction lui avait procuré :

Je revivais ces semaines passées dans l'antique palais délabré dont j'avais fait le palais Altinengo de l'Entrevue, où j'avais décrit minutieusement les vastes pièces aux stucs peints ou dorés, au pavimento de mosaïque incrusté de fragments de nacre, à la singulière porte en miroirs. Certes, je n'y avais pas vu apparaître le visiteur mystérieux en «tabaro e baüta » mais c'était là que son existence s'était formée en mon esprit et qu'il avait pris corps en mon imagination [...]. De ces divers contes, ce dernier est peut-être celui que je préfère; et si je le préfère, c'est moins à cause de ce que je puis penser de sa valeur littéraire et de sa qualité d'invention que parce qu'il me rend présentes des heures particulièrement précieuses à mon souvenir, parce qu'il me rapproche de cette Venise que j'ai tant aimée et où je suis revenu si souvent chercher un délassement et un repos². 
Ce n'est qu'en 1924 qu'il y retournera. Un nouveau livre en sortira: L'Altana ou la Vie vénitienne (1899-1924): il s'y attachera à "relier le passé au présent, la Venise si longuement, si minutieusement souvenue, à la Venise aujourd'hui retrouvée ${ }^{3}$ ».

2 Ces retrouvailles heureuses étaient également au programme du héros de L'Entrevue qu'une profonde crise sentimentale, suivie d'une dépression nerveuse, avait séparé, trois ans durant, de la ville où il séjournait régulièrement, à l'automne, depuis une quinzaine d'années. Cependant, une série de circonstances feront qu'au lieu de remettre ses pas dans la vie heureuse qui y avait été la sienne, il sera contraint de renoncer à sa location habituelle pour habiter le mezzanino (l'entresol) du palais Altinengo; des crises d'anxiété récurrentes se produisant en outre lors de ses promenades en ville ou sur la lagune, et ce palais s'avérant le seul endroit de la ville où il trouve la tranquillité qu'il était venu chercher, il prendra la décision de s'y confiner dans la pièce qu'évoque la préface plus haut citée. Il en résultera pour lui une aventure que l'auteur évite de qualifier de façon univoque, oscillant entre plusieurs qualifications génériques: "fantasmagorie», "policier», «fantastique», « merveilleux » : une aventure en somme aussi « incertaine » qu'imprévisible, à l'instar de Venise ${ }^{4}$. Pour autant, si le héros s'y trouve bien confronté à l'étrange, au mystère, à l'inexplicable, ce ne sera jamais une occasion d'épouvante mais tout au plus de surprise ou d'étonnement, jusqu'à ce que la suite des événements lui offre le bonheur émerveillé de pénétrer dans une vie exceptionnelle à laquelle rien ne le prédestinait.

3 La principale singularité de ce conte, dans le contexte de ce que Sophie Basch nomme la «folie vénitienne» du roman de l'époque ${ }^{5}$, c'est que l'essentiel de la symbolique de la cité ancienne et actuelle se trouve analogiquement relié au palais, tout particulièrement au salon du mezzanino où vit le héros, par tout un réseau d'intersignes très subtilement tissé; de sorte qu'au fil de la progression narrative, ce salon devient une sorte d'accumulateur où vient se concentrer, dans le présent, la charge proprement magnétique de la Venise du settecento qui revient, pour ainsi dire, s'y matérialiser : d'abord dans l'imaginaire mémoriel du héros à propos de la disparition d'un buste de cette époque dont il a conservé une parfaite image mentale, puis à la faveur de la découverte inopinée d'un portrait de l'ancien propriétaire du palais, enfin à travers la manifestation de l'apparence de ce dernier de l'autre côté du haut miroir en forme de porte factice qui occupe l'un des murs de la pièce. Une autre singularité serait qu'à la différence du personnel cosmopolite et mondain de la plupart des récits "vénitiens » de cette époque, le héros-narrateur de celui-ci s'auto-définit comme une sorte d'homme sans qualités, très peu remarquable et content de l'être. Le moins singulier de l'aventure néanmoins exceptionnelle qui le requerra, ce sont les thèmes du miroir et du double qui hantent le symbolisme français ${ }^{6}$ et Régnier en particulier ${ }^{7}$, mais aussi l'imaginaire fantastique et merveilleux bien étudié notamment par Max Milner ${ }^{8}$ ou, en ce qui concerne leurs racines anciennes, par Jurgis Baltrusaitis 9 . Pour autant, on espère montrer que, dans ce domaine également, la rigueur proprement poétique avec laquelle l'auteur a incrusté ces thèmes en la textualité même de son récit, n'est pas le moindre de ses mérites.

\section{Mosaïque}

Ayant donc trouvé occupé le logis où il comptait demeurer comme par le passé, le héros s'en est ouvert à l'un de ses anciens amis qui lui a donné l'adresse du palais Altinengo. 
Le jour de la visite, la propriétaire l'ayant conduit devant la porte du mezzanino qu'elle loue, il y est frappé par un détail du pavement : «Parmi les petits cubes de marbre qui formaient ce pavimento, un fragment de nacre était incrusté. Cette bizarrerie m'intéressa ${ }^{10} »(60)$. Un peu plus tard, alors qu'il a visité le vestibule et deux chambres tellement délabrés qu'il s'apprête à renoncer à cette location, il découvre avec émerveillement un salon rococo bien conservé dont l'attrait irrésistible le fait changer d'avis. Entre autres merveilles, le même détail s'y reproduit: "Le pavimento s'incrustait, çà et là, de fragments de nacre » (63). Plus tard encore, ayant emménagé au palais, il médite sur ce que l'isolement et le silence du lieu comportent de favorable pour l'obtention de la tranquillité d'âme qu'il recherche à Venise :

Et j'imaginais, non sans plaisir, de lentes journées et de lentes soirées dans le salon de stuc aux arabesques ingénieuses et aux chinoiseries chimériques, où les grands feux d'automne et la lumière des bougies illumineraient de leurs reflets tremblants les figures et les pagodes des panneaux de faïence, éclaireraient les volutes des moulures et les anfractuosités des rocailles et feraient briller doucement les énigmatiques fragments de nacre qu'une fantaisie inexplicable avait incrustés dans la mosaïque du pavimento. (87)

Ainsi revient l'attestation de ce détail, déjà qualifié de "bizarrerie » selon une isotopie de l'énigme qui circule dans le conte du début à la fin. Lors de la première occurrence de ce motif, il avait été décrit, quelques lignes plus bas, comme « menu disque de nacre posé à son seuil comme une mouche de lune à quelque visage vénitien d'autrefois " (60). Il s'agit là d'une analogie classique dans sa structure ${ }^{11}$ : ce disque est au seuil du mezzanino ce qu'une mouche en forme de lune est à un ancien visage vénitien. Un visage du settecento, époque dont atteste évidemment l'esprit de la décoration du salon.

Une fois installé, le héros passera le plus clair de son temps à rêvasser dans ce salon illuminé de grandes flambées qui réchauffent l'espace de ce palais délabré où les fenêtres sont loin d'être hermétiques; c'est surtout au soir, quand les bougies sont allumées, que tout son charme se déploie : « Les vieilles dorures s'animaient et toute la pièce s'emplissait d'une atmosphère de luxe mystérieux. Dans la mosaïque du pavage, les fragments de nacre luisaient doucement de phosphorescences marines" (116). L'analogie implicitement suggérée est alors celle-ci : le pavage du salon est à la mer qui baigne Venise, ce que la luisance des disques de nacre est aux lumières marines crépusculaires ou nocturnes. Or ces lumières apparaissent également dans un autre élément $\mathrm{du}$ décor de la pièce, laquelle comporte trois portes semblables à celle du vestibule, mais dont le quatrième mur est occupé par un haut miroir encadré de marbre jaune, sorte de « porte factice » (111) répondant aux trois autres par ses dimensions :

Avec le temps, il avait acquis un indéfinissable et admirable aspect d'eau profonde et comme souterraine, et les images qui s'y formaient y prenaient une sorte d'obscurité crépusculaire, quelque chose de lointain et de mystérieux. Les lumières s'y reflétaient comme voilées. Tout y apparaissait grave et distant dans un recul d'un au-delà extraordinaire. (111)

Par une double analogie, la profondeur maritime transparaît donc dans l'eau de ce miroir simulant une porte, autant que dans la nacre du pavage avec la même qualité de luminosité en clair-obscur. Mais la porte-miroir ${ }^{12}$ a une autre propriété : celle de rédupliquer le salon à l'envers : "Elle se dressait comme un portique ouvert sur un autre monde et, dans un songe réel, on y apercevait, en une perspective inverse, ce même salon de stuc, avec ses mêmes arabesques et ses mêmes figurines, mais situé dans un lointain séculaire, dans un recul inaccessible et mystérieusement nocturne » (88); de sorte que le héros, lors de longues heures de rêverie assis dans un fauteuil rococo et 
accoudé à une table laquée, se complaît à retrouver dans ce miroir « le décor fastueux et mélancolique où survivait la grâce de la vieille Venise de jadis et de ses intimes fantaisies » (92). À l'inverse de la perception d'Alice lorsqu'ayant pénétré de l'autre côté du miroir, elle qualifie de "vieille » la pièce qu'elle vient de quitter ${ }^{13}$, c'est la Venise ancienne, celle du settecento qui hante l'autre côté de la porte-miroir.

Dans l'avant-dernière des onze séquences qui composent le récit, tout ce dispositif analogique qui tend à identifier la Cité ancienne au salon du palais Altinengo vient en quelque sorte se mettre en abyme ou se condenser, à la veille de l'événement qui met un terme définitif au séjour du héros en ces lieux. L'hiver approche, l'époque des grandes marées, des tempêtes et des inondations est arrivée; depuis quelque temps, le héros a constaté, puis vérifié, qu'à l'heure des bougies son reflet s'absente du miroir, sans d'ailleurs s'en émouvoir autrement que pour se réjouir qu'un tel destin lui soit assigné ; ensuite de quoi s'est matérialisé à sa place le fantôme du propriétaire vénitien des lieux au xvIII ${ }^{\mathrm{e}}$ siècle, lequel se déplace comme chez lui de l'autre côté du miroir, jusqu'à la nuit où il découvre qu'il n'est pas seul; d'où finit par naître une passion commune de se rencontrer qui fait de chacun la hantise de l'autre, se contemplant, immobiles, dans l'attente de l'événement qui doit survenir :

[...] tandis que derrière nous, à la clarté des bougies, le décor magnifique et baroque des vieux stucs et des faïences luisait de ses ors miroitants ; tandis que, au-dessus de nos têtes, s'étageait sur ses pilotis rongés l'antique palais des Altinengo en sa vétusté branlante; tandis qu'alentour, la mystérieuse Venise nocturne se superposait, fragile, compliquée et merveilleuse, à son propre reflet, doublée par le miroir de sa lagune circulaire et des eaux insinuées, par mille canaux, en sa mosaïque architecturale, Venise sur qui brillait, semblable à l'un des fragments de nacre du pavimento, le disque écorné de quelque lune étincelante [...]. (128-129)

La mosaïque du pavage s'avère ainsi l'analogon de la ville tout entière en "sa mosaïque architecturale » infiltrée de canaux, tandis que la réduplication dans le haut miroir rectangulaire du salon illuminé rime avec le dédoublement de Venise au miroir circulaire de sa lagune; que la ruine menaçant le vieux palais dans ses fondements comme dans ses étages supérieurs, fait écho à la fragilité redoublée, à l'époque de l'Acqua alta, d'une architecture urbaine aussi fragile que séculaire; - et qu'enfin, last but not least, tel fragment nacré éclaire tel pavage comme l'astre des nuits la cité des eaux. Un astre « écorné » cette nuit-là comme va s'avérer « fêlée » (136) la tête du héros par la chute de la porte-miroir cette nuit-là, comme il l'apprendra en sortant du coma, de la bouche du médecin qui l'a soigné, d'où s'ensuivit en bonne logique analogique que

non seulement la porte qui vous a blessé a dégringolé, mais, le lendemain de votre accident, une partie du mur s'est écroulée et le pavimento s'est effondré. Le palais était, quand vous y êtes entré, dans un état de délabrement inquiétant et n'a pas résisté à la grande marée et au vent qui a soufflé en tempête. Il a bien failli s'abattre tout entier dans le canal. D'ailleurs, la municipalité l'a fait évacuer et il est question de le démolir. (133-134)

Régnier a probablement joué là avec La chute de la maison Usher où la fissure presque invisible qui relie en zigzag, au début de la nouvelle, la muraille du château à l'eau noire de l'étang qui le baigne, est devenue lors de la nuit de tempête finale sous une lune rouge-sang, la faille qui, divisant en deux l'édifice, le précipite dans l'étang.

Au reste, l'écroulement du palais avait été envisagé d'emblée par le héros lors de son installation ; c'était même une donnée de son attrait :

Venise est la ville du monde où l'on voit le plus de maisons penchées, déjetées, moribondes et qui semblent ne tenir debout que par un miracle d'équilibre. Ce miracle, le palais Altinengo le réalisait depuis longtemps sans doute et le réaliserait 
sans doute longtemps encore, sous la surveillance d'une édilité discrète, mais avisée. Et puis, qu'importait après tout! Ce risque me laissait assez indifférent. Qu'était-il pour quelqu'un qui faisait peu de cas de la vie et qui ne lui demandait, en somme, que le repos et l'oubli ! (86)

Mais si cette isotopie du délabrement menaçant ruine est clairement développée tout au long du récit, celle de la fêlure est seulement insinuée en certains détails ; ainsi ce palais Altinengo ai Carmini signalé au héros par un ami comme devant bien lui convenir, et dont il ignorait l'existence alors qu'il est proche de l'église des Carmini qu'il connaît en revanche fort bien - ce palais compte au nombre de ses charmes le plaisir de l'écoute des cloches vénitiennes :

Ce jour-là, tout se passa donc comme de coutume. [...] L'air devait être pur, car les cloches des Carmini et des églises voisines tintaient avec une sonorité vive et nette. Je m'amusais à les reconnaître à leurs timbres. Je distinguais celles de San Sebastiano et celles des Frari, celles de l'Arcangelo Rafaele » (113-114).

Une exception néanmoins: "Les cloches des Carmini, dont le son est un peu fêlé, sonnaient si près que je ne leur donnais plus aucune attention » (114). Nul doute, dans l'économie du récit que cette sonnerie fêlée n'annonce ironiquement, dans le langage de la Cité, telle inéluctable fêlure de crâne, d'ailleurs redoublée in fine par une autre rime que nous aurons à envisager plus loin, pour l'énigme de sa fonction dans l'économie générale de l'intrigue. En effet, comme le diable, c'est dans les détails que gît le « démon de l'Analogie » car, après tout, quel est donc le double anagrammatique du mot « crâne », si ce n'est le mot « nacre »?

\section{S'oublier?}

En tout état de cause, le héros du conte se trouve donc la proie consentante de la Venise du settecento. Était-ce son projet, en y retournant après trois ans d'oubli de nombreuses années de séjours heureux au contact d'excellents amis qui l'avaient, comme ils disaient "vénitianisé " (15) ? La réponse est négative. Si ce Parisien est revenu à Venise après la parenthèse d'une grave crise sentimentale culminant dans une grave maladie qui l'a laissé très affaibli physiquement et moralement, c'est pour tenter d'y retrouver l'atmosphère mentale antérieure à cette crise, autrement dit une mémoire heureuse capable de contrebattre les souvenirs rémanents d'une souffrance morale dont rien ne sera dit, puisque ce récit autodiégétique entend se borner aux « faits » concernant ce dernier séjour :

Mais avant d'arriver aux circonstances qui m'amenèrent à être, durant plusieurs mois, l'hôte de cette étrange demeure, il est nécessaire que je dise quelque chose des raisons qui, en cette fin de septembre $189 . . .$, me déterminèrent à reprendre une fois de plus le chemin de la Cité bien-aimée.

Sur ces motifs, je serai bref, car ce n'est pas une « confession » que j'entreprends ici. J'ai toujours répugné aux confidences, ne me jugeant pas assez intéressant pour solliciter sur moi-même l'attention d'autrui. (8-9)

Dans le prologue dont est tiré ce passage, le héros-narrateur atteste de ce que son intérêt pour cette cité n'a rien à voir avec ce que la littérature et le tourisme mondain fin-de-siècle montent en épingle : mythe de la décadence, esthétisme plus ou moins compétent, goût du passé par principe, affichage de soi, etc. :

Oui, et je tiens à le bien établir, mon amour pour Venise fut toujours un amour sain et simple, un amour familier, exempt de snobisme et d'esthétisme, exempt aussi de romantisme [...]. Jamais je ne me suis cru obligé de vivre à Venise "autrement " qu'ailleurs, dans une exaltation particulière et dans un état d'esprit inaccoutumé. 
Jamais je ne m'y suis attendu à des impressions exceptionnelles [...]. Je ne suis pas de ceux que Venise a ensorcelés par avance et au doigt de qui elle a passé son anneau magique, et je ne me suis jamais drapé dans le manteau du romantisme vénitien. (11-12)

Bien informé, cultivé, capable d'apprécier et de goûter, non dépourvu de lectures, etc., en ce qui concerne la Venise ancienne, ce qui l'a néanmoins toujours intéressé jusqu'alors, c'est son présent : «Venise me suffit en elle-même et je n'ai pas besoin de son passé pour subir le charme de son vivant enchantement » (10-11). Enfin, il n'est a priori pas plus concerné, sinon à titre de curiosité amusante, par sa légende occulte telle que transmise par le Casanova des « pyramides » chiffrées ou les chapitres des Mémoires de Gozzi relatifs à sa persécution par les esprits pour les avoir mis en scène dans ses comédies (33). S'il écoute, sans guère broncher, son vieil ami Prentinaglia prétendre que cette Venise-là réémerge à leur époque comme en attestent certains faits, il demeure foncièrement sceptique à cet égard, considérant ses propos comme des « jeux fantasmagoriques » (78), sinon comme une de ces tentatives de mystification dont le sieur est coutumier, ne le provoquant sur ce terrain que parce qu'il le sait « incrédule».

Ce qu'il est venu rechercher, c'est donc une thérapeutique de l'adoucissement, voire de l'effacement du souffrir, par une culture retrouvée de la fréquentation de cette cité tant aimée naguère en compagnie d'amis depuis défunts, puis en solitaire habitué d'une pension tenue par deux sœurs attentives :

Je revis en pensée le cyprès de l'étroit jardin de la Casa Trigiani, ses sauges écarlates. Je réentendis les voix criardes et amicales des Sorelle, le bruit des socques martelant les dalles des Fondamenta Barbaro, le cri des marchands ambulants, le « staï » du gondolier tournant à l'angle du petit rio, toutes les rumeurs familières de la Venise populaire, et, dans le ciel, les belles cloches de la Salute et des Gesuati. Ma décision était prise. Il ne me restait plus qu'à télégraphier aux Sorelle Trigiani la date de mon arrivée. (17-18)

Pour autant, le lecteur est frappé de ce que ce projet rencontre ab initio d'étranges obstacles indépendants de la volonté du héros; c'est ainsi que le télégramme une fois rédigé se retrouve plié dans son portefeuille, alors qu'il est déjà dans le train, cependant qu'il se souvient parfaitement l'avoir remis à un domestique pour qu'il fasse le nécessaire. Une distraction ou un acte manqué qui le mène à s'interroger sur la précipitation d'un départ qu'il pouvait fort bien différer eu égard à la réalité médicale de son état. Une fois parvenu à destination, il devra affronter un second discord avec ses intentions, en constatant que les Sorelle ont depuis trois mois quitté Venise, après avoir loué leur Casa. Qu'il eût donc envoyé ou non le télégramme n'eût rien changé en somme à la situation : il va lui falloir louer ailleurs, ce qui l'inquiète car la tranquillité à Venise n'est pas si commode à obtenir.

Un autre symptôme de la difficulté qu'il va rencontrer à tous moments pour retrouver, en ce séjour, l'équivalent de ce que sa mémoire lui rappelle des heureux précédents d'avant sa crise, c'est qu'à peine sorti du train, et embarqué sur la gondole qui le conduit à la ville, ce n'est pas la Venise présente qui le requiert, mais une songerie sur ses bonheurs passés qui l'absente de son regard:

Cette approche de la ville aimée provoquait toujours en moi une impression de plaisir, indéfinissable, mais profond... Si, cette fois, je n'éprouvai pas ce plaisir dans sa plénitude, je n'en ressentis pas moins une réelle satisfaction lorsque, descendu $\mathrm{du}$ train et sorti de la gare, je vis l'eau du canal baigner les marches du quai et, audessus des fers des gondoles rangées, s'arrondir dans le ciel le dôme vert-de-grisé de S. Simeone. Soudain, toute la Venise de jadis revivait dans mon souvenir et il me sembla, lorsque la rame battit l'eau et que la gondole qui m'emportait vira 
doucement, que je laissais derrière moi ma pesante et douloureuse vie d'hier et que je n'étais plus qu'une ombre allégée qui s'en allait dans le silence et la lumière, vers la paix, le calme et l'oubli.

Ces pensées m'occupèrent assez pour que je demeurasse presque indifférent au doux spectacle de Venise retrouvée. (20-21)

Cette indifférence s'avérera une constante au fil du récit, la cité se dérobant de fait à toutes ses tentatives pour la retrouver telle qu'il l'avait connue. Ainsi, dès le soir de son arrivée, après une installation et un dîner expédiés à tel hôtel élu sans savoir pourquoi, le voici entreprenant une de ces balades dans la Venise nocturne dont il avait été coutumier :

Que de fois, en effet, ne m'étais-je pas aventuré dans l'obscur et capricieux dédale vénitien! J'étais arrivé à le connaitre si parfaitement que je m'y dirigeais avec une certitude presque absolue. Or, ce soir-là, je m'aperçus bientôt que je ne possédais plus mon ordinaire sécurité d'orientation. À plusieurs reprises, je fus obligé de m'arrêter, incertain de la direction suivie et même, une fois, je m'engageai dans un de ces « rami » sans issue et qui aboutissent à un " rio " devant lequel on est forcé de rebrousser chemin. (23)

Cette difficulté se redouble de l'émergence d'un état de "nervosité » aux causes peu définissables, qui se développe en "malaise », puis en " appréhension », enfin en une " angoisse sournoise " quoique sans cause, puisque le héros sait de longue expérience que les nuits vénitiennes sont sûres (25). Il expérimente là, pour la première fois de sa vie, l'effet que produiront immanquablement sur lui toutes ses sorties vénitiennes dès qu'il s'éloignera un tant soit peu, soit pour telle promenade sur la lagune, soit simplement pour déjeuner ou dîner, du palais Altinengo, au point qu'il se résoudra à n'en plus sortir. C'est que, sous couvert des réminiscences bénéfiques qu'il entendait tirer de ce voyage, ce qu'il recherche fondamentalement ce n'est pas d'abord la mémoire, mais l'amnésie « pour quelqu'un qui faisait peu de cas de la vie et qui ne lui demandait, en somme, que le repos et l'oubli » (86).

Cette aspiration à l'oubli, qui implique celui de soi, est une donnée caractéristique du personnage tel qu'il se dépeint dans le prologue de son récit, soit comme un anonyme heureux de son état, transparent au regard d'autrui : "Je suis un homme des plus ordinaires et qui ne se distingue du commun par aucune capacité spéciale, ni par aucun mérite intellectuel qui ait de quoi le mettre en vue. J'ai toujours vécu pour moi-même et j'ai toujours trouvé tout naturel de passer inaperçu aux yeux des autres » (9). Cette vocation spontanée au détachement trouve dans le palais Altinengo un site de prédilection parfaitement accordé, comme l'annonce le premier contact du héros avec le haut miroir-porte du salon :

[...] je me sentais étreint d'une émotion indéfinissable. Ne serais-je pas un intrus dans ces lieux? N'allais-je pas troubler leur abandon? De quel droit, après tout, osais-je m'introduire en leur silencieux mystère ? Hélas, en tout cas, je ne serais pas un intrus bien gênant! Je n'y apporterais avec moi ni la joie bruyante de la jeunesse, ni les rires de la santé. Je faisais si peu partie de la vie; et cependant, c'était pour essayer de revivre que je revenais dans cette Venise, chercher dans mon passé de quoi y rattacher ma misère présente! Vaine tentative, chimérique espoir. C'était à peine un vivant qu'accueillerait dans sa paix à-demi morte ce vieux palais Altinengo. Et soudain, dans la grande glace, debout en son cadre de marbre, je m'aperçus lointain et vague, comme si mon image fût soudain entrée dans la région reflétée et muette des ombres .... (66)

- Une région dont Chateaubriand affirme, après Virgile, qu'il faut disposer du rameau d'or pour qu'elle consente à s'ouvrir à l'impétrant ${ }^{14}$. 
Mais qu'importe à cette heure du récit: celui qui s'envisage comme à peine vivant ressent comme un bonheur inattendu sa concordance avec ce palais qui tient à peine debout et dans le mezzanino duquel il s'abandonne, dans une solitude quasi absolue hormis les services ancillaires de sa logeuse, à des « rêvasseries » continuelles qui lui procurent, auprès de la cheminée, l'oubli du souci dans l'oubli du temps : «De longues bûches déposées sur le pavimento me fournissaient de quoi l'alimenter pendant la journée et une partie de la nuit, car je prolongeais mes veilles assez tard. Mon temps se passait à une sorte de rêverie indéfinie où j'oubliais le cours des heures » (105).

\section{D'une économie symbolique}

La poétique du détail caractéristique de l'art de Régnier inclut le monde des couleurs. C'est ainsi une certaine qualité de jaune doré ${ }^{15}$ qui caractérise ce salon où le héros vit, quasi confiné, jusqu'à la chute du récit :

C'était une sorte de salon, à peu près carré, à deux fenêtres, entre lesquelles une cheminée de marbre jaune se dressait, surmontée d'un miroir à volutes dorées. Le ton du marbre et des vieux ors se complétait par la couleur des murs. Ils étaient peints d'une couleur jaune, d'un jaune délicieux, ambré comme du miel, et, sur ce fond, d'une exquise et molle douceur de teintes, se détachaient en blanc des moulures de stuc formant des arabesques symétriques. Ces arabesques, d'un dessin et d'une fantaisie admirables, sur chacun des trois côtés de la pièce, encadraient de grands panneaux de faïence blanche où étaient figurées, en or et en noir, des scènes de chinoiseries. À droite et à gauche de chacun de ces tableaux, deux autres plus petits en leurs mêmes cadres de stuc. Au plafond, le même décor de chinoiseries se continuait et se complétait à la fresque par des oiseaux, des fleurs et des insectes. Le pavimento s'incrustait, çà et là, de fragments de nacre et, à l'un des angles du salon, une haute glace, dans un cadre de marbre jaune, debout, le reflétait en toute sa fantaisie somptueuse et saugrenue, en tout son mystère imprévu et charmant. (62-63)

Parmi les meubles qu'il achète chez l'antiquaire Zotarelli avant d'emménager, il a choisi, en harmonie avec cette tonalité et ces motifs chinois, une grande table laquée jaune elle aussi ornée de chinoiseries or sur noir. Ce motif chinois, caractéristique de la Venise du settecento, rime dans le récit avec un personnage vénitien que le héros fréquentait régulièrement lors de ses séjours antérieurs : il s'agit du Chinois peint dans le salon oriental du café Florian où il avait l'habitude de se retrouver naguère, place Saint-Marc, avec ses amis Tiberio Prentinaglia, Lord Sperling et Otto de Hohenberg; c'est d'ailleurs "sous le Chinois » qu'il vient s'asseoir à l'issue de son errance du premier soir dans le dédale vénitien, sa place ordinaire se trouvant libre ce soir-là. Il ne tardera guère à voir apparaître Prentinaglia, dont nous apprenons qu'il a "le visage long et jaune »; c'est ce dernier qui l'orientera vers le palais du salon jaune, dont la propriétaire est ainsi présentée: "C'était une femme d'une soixantaine d'années, courte, trapue, vêtue de noir, le visage carré, avec des yeux enfoncés, un teint jaune, des cheveux gris » (57). C'est elle qui assure son service, d'ailleurs fort discrètement. Lors d'une promenade en gondole sur la lagune morte, le lendemain de son arrivée, le héros sera saisi d'une nouvelle crise d'angoisse à l'approche d'un bâtiment connu des vénitiens sous le nom de Casa degli Spiriti, dont la réputation sulfureuse s'inscrit dans ce nom, lequel s'accorde avec les préoccupations de son nouveau propriétaire, qui n'est autre que son ami Lord Sperling passionné d'occultisme et de magnétisme; outre d'autres traits sur lesquels il faudra revenir, ce bâtiment est également relié au salon du 
palais Altinengo par sa couleur : «L'extérieur n'en avait pas été modifié ; la façade était toujours badigeonnée du même crépi jaune » (76). C'est également la couleur de la clinique du docteur Bellincionni où se réveille à la fin du récit, comme le lui apprend l'infirmière; elle fait également partie de ses souvenirs: «Plus d'une fois, en me promenant dans la Giudecca, j'avais passé devant la petite maison jaune aux grandes cheminées en hottes qui montrait, inscrite au-dessus de la porte, une grande croix rouge. Une fois même, j'étais entré pour examiner le jardin que l'on apercevait du dehors, à travers le vestibule » (132).

Un autre secteur de cet ensemble d'îles qu'est Venise en sa lagune se trouve relié au salon du palais Altinengo par la nécessité du chauffage : il s'agit de l'île de Murano, dont le héros fait venir les grandes bûches qui alimenteront la cheminée :

Les verriers de Murano se servent, pour alimenter leurs fournaises, de longues et fortes bûches qui viennent de l'Alpe voisine. La dimension de la haute cheminée de marbre jaune en permettait l'usage. Elles y brûlaient en flammes pétillantes et en braises précieuses comme des pierreries. Ce luxe du feu s'alliait merveilleusement avec le luxe doré des stucs, des faïences et des rocailles, et il s'en répandait par toute l'atmosphère une sorte de tiède bien-être que j'appréciai fort, dans l'était presque maladif où je me trouvais. (103)

La célèbre verrerie de Murano est encore présente dans le salon par un autre élément décoratif qu'il a souhaité: "des appliques en verre fumé de Murano»(82) qui s'illuminent chaque soir "à l'heure des bougies ", tandis que flambe la cheminée. Ainsi s'accorde la tonalité générale de « miel doré » (64) qui a immédiatement séduit le héros alors qu'il découvrait ce salon, avec les transparences claires-obscures des appliques, tandis que dans la cheminée de marbre jaune, domine le rougeoiement des flammes: «Les bougies brûlaient dans leurs appliques, le feu rougeoyait dans la cheminée » (116). Cette note rouge se trouve elle-même reliée à tout un réseau de signes disposés du début à la fin du récit, nous y reviendrons plus loin.

Outre la couleur de ses murs, d'autres éléments relient la propriété de son ancien ami Lord Sperling (qu'il ne rencontrera pas au cours du récit) au mezzanino du palais Altinengo. Ainsi, comme le salon du palais, la Casa forme un carré ${ }^{16}$. Par ailleurs, comme le mezzanino, elle s'agrémente d'un jardin ; une fois le marché conclu, la signora Verana conduit son locataire à la chambre où elle demeure habituellement et qu'elle lui abandonnera durant le temps de son séjour :

Hors cette chambre, à peu près habitable, toute cette partie du palais était dans un état de délabrement extrême et vraiment semblait menacer ruine. Mon domaine se terminait par une assez grande salle d'où un escalier extérieur donnait accès à un jardin planté de légumes et de quelques arbres. Au bout de ce jardin se dressait une construction bizarre, sorte de petit temple à colonnes et à fronton : l'ancien casino du palais. (65)

Les nobles vénitiens avaient en effet coutume de réserver aux jeux « tant du hasard que de l'amour " écrit Régnier ${ }^{17}$, un bâtiment spécifiquement dévolu à cet usage hors de leur palais. Or la Casa degli Spiriti est un ancien casino ; cela, nous l'apprenons en lisant L'Altana, un récit autobiographique ultérieur où Régnier retrouve Venise après la guerre. Il y a notamment demeuré au palais Vendramin ai Carmini dont le salon lui a fourni le modèle inspirant celui de L'Entrevue, et en ce qui concerne la Casa degli Spiriti, nous y lisons ceci :

Était-il sorti [un ivrogne observé au café Florian] de ce Casino degli Spiriti qui se dresse au bord de la Lagune non loin de la Madonna dell'orto et que l'on prétend hanté par les esprits ? [...] Que de fois, de la Lagune, je l'ai regardée, cette grande 
bâtisse carrée aux murs jaunes qui se dresse non loin de la Madonna dell'Orto et que l'on disait hantée, ce qui lui valait d'être appelée la Casa degli Spiriti ${ }^{18}$.

L'Entrevue nous apprend que cet ancien casino est muni d'un jardin qui, avant l'emménagement de Lord Sperling, se souvient le héros, «donnait sur la lagune, par une terrasse jadis à l'abandon", et qui montre désormais, observe-t-il, "des arbres soigneusement taillés et des buissons de roses qui, débordant la balustrade, pendaient presque jusque sur l'eau » (77). Le dispositif jardin-casino ancien est donc commun au mezzanino du palais Altinengo et à la Casa degli Spiriti, ils se correspondent en somme comme le modèle réduit à l'original ${ }^{19}$. Deux autres éléments les relient encore dans l'économie symbolique du conte. En se procurant les meubles dont il a besoin auprès d'un antiquaire qu'il a bien connu naguère, un homme aussi jovial que serviable ordinairement, le héros constate aussitôt sa discrète réticence à l'égard du lieu comme de sa propriétaire. Il tente d'en profiter pour en savoir un peu plus sur la signora Verana et finit par apprendre qu'elle a longtemps été au service de Lord Sperling à la Casa degli Spiriti, jouant auprès de lui le rôle de "gouvernante » qu'elle jouera auprès de son propre locataire (64); en outre, et c'est le second élément, une restauration du palais Altinengo avait également été envisagée mais il a fallu y renoncer, l'antiquaire demeurant dans le vague sur certaines raisons l'ayant empêché : « On dit qu'elle aurait voulu ouvrir une pension de famille au palais Altinengo, mais le palais est mal situé et il faudrait trop de réparations. Et puis, et puis... Alors vous n'avez pas besoin d'autre chose? » (70-71). Cette énigme, comme bien d'autres dans le récit, demeurera en suspens ; quant au petit temple-casino, quoique le héros doive parcourir le jardin lors d'une exploration qui s'avérera décisive, jamais il ne songera à y pénétrer.

Si Venise est, dans la symbolique analogique du conte, une "mosaïque architecturale » structurée par le réseau complexe de ses canaux (129), et si les arabesques de stuc du salon jaune, reflétées dans l'eau de son haut miroir à la lumière des flammes, mettent en abyme cet ensemble, les acteurs présents et passés de l'intrigue se trouvent non moins reliés par de discrets canaux. Nous savons que Prentinaglia et Sperling sont de vieilles connaissances du héros, qui découvre, lors de son premier contact avec sa future hôtesse, qu'elle connaît et respecte le premier: «Elle semblait méfiante et taciturne, et me considérait avec une curiosité sans bienveillance. Cependant, au nom de Prentinaglia, sa figure s'éclaira d'une espèce de sourire et elle esquissa une manière de révérence qui n'était pas sans dignité » (57). Ce sera l'un des sujets de méditation du héros, que de s'interroger sur la nature des liens qui unissent ce trio ; réfléchissant aux expériences de Sperling, qui est un disciple de William Crookes, ce membre de la Royal Society, à l'époque aussi réputé que controversé pour avoir appliqué les techniques de la psychologie expérimentale aux phénomènes parapsychiques ${ }^{20}$. Il se demande donc

si cette signora Verana que Zotarelli m'avait dit avoir été au service de Sperling, n'avait pas joué un rôle dans les expériences psychiques dudit Sperling et si, après tout, l'insolite disparition du petit buste du Musée civique n'était pas sans corrélation avec les jeux fantasmagoriques dont le superstitieux Prentinaglia m'avait signalé l'existence, lors de notre rencontre au café Florian. (78)

\section{Ambiguiité générique}

19 Cette disparition constitue l'énigme motrice du récit, une énigme qui sera traitée parallèlement sous trois régimes génériques, sans préjudice de leurs nuances : celui du roman d'enquête policière, celui du récit fantastique, celui du conte merveilleux. Deux 
de ces régimes s'affrontent d'emblée lors du récit qu'en procure Prentinaglia au narrateur, dans une version concluant sans appel à l'intervention du surnaturel, en dépit de l'incrédulité sceptique de son interlocuteur. Un petit buste a donc disparu de sa vitrine au Musée civique, sans qu'aucune trace d'effraction ait pu être constatée : «Et ne m'alléguez pas que l'affaire du buste est d'ordre naturel et qu'elle s'éclaircira d'elle-même un beau jour... Non, l'enquête a été conduite minutieusement, mais elle n'a donné aucun résultat » (41). De ce buste, le narrateur se souvient tout à fait bien :

J'avais souvent admiré ce précieux bibelot, qui m'avait frappé par sa qualité artistique. L'homme représenté, quelque patricien de Venise sans doute, attirait nécessairement l'attention. Sa figure était étroite, maigre, distinguée, avec un nez long et une bouche sensuelle. Tout dans ce personnage disait le voluptueux et l'amoureux. Il avait dû aimer passionnément la parure, la table, les fleurs, les femmes, mais il y avait aussi sur ce visage l'expression d'une insatiable curiosité. De quoi avait-il été si curieux, ce seigneur vénitien : des secrets de son cœur ou des secrets de l'État? Que de finesse dans cette physionomie attentive et ardente! Et quelle vie avait-il vécue? Quelles aventures avaient été les siennes? Quel nom avait-il porté ? Plus d'une fois j'avais interrogé Prentinaglia sur l'origine de ce buste. Prentinaglia, je me le rappelais fort bien, s'en était enquis auprès du directeur du Musée, mais on n'avait pu le renseigner. On ne savait plus à quelle époque le buste était entré dans les collections. La fiche le concernant avait été sans doute égarée. Le catalogue ne portait aucune indication. Tout ce que l'on avait pu dire, c'était que l'objet figurait depuis longtemps dans les vitrines. Quant à l'identité $\mathrm{du}$ personnage, même ignorance. L'inconnu semblait s'en amuser en son énigmatique et fin sourire. Tous ces détails me revenaient à l'esprit avec l'interrogation de Prentinaglia. (39-40)

$\mathrm{Au}$ sein du réseau analogique tissé par le récit, la physionomie du personnage représenté s'apparente à un certain nombre d'autres. Ainsi du Chinois sous l'image duquel cette disparition est contée, dont la «bonhomie narquoise " retrouvée par le héros ce soir-là (28) rime avec "l'énigmatique et fin sourire » du buste disparu - l'adjectif «narquois» qualifiant d'ailleurs dans ce qui s'ensuivra (56 et 93), la physionomie de son modèle, dont la semblance finira par apparaître au héros dans son portrait peint retrouvé au mur d'un réduit du mezzanino, puis lorsqu'il surgira, en pied cette fois, de l'autre côté du miroir de son salon, enfin lors de l'adieu du narrateur à son apparition, dans l'explicit du récit: «[...] je saluai une dernière fois son image qui semblait, de son sourire énigmatique, narquois et mélancolique, répondre à mon salut, et que je n'ai plus jamais revue!». Mais cette physionomie ressemble aussi par certains traits à celle de Prentinaglia, lequel se trouve doté, à son instar, d'un " grand nez » et dont la bouche "mince et sinueuse " (30) rime grossièrement avec "la bouche fine" dont, lors d'une ses remémorations ultérieures (56), le héros pourvoit le buste et/ou son modèle.

Si ce buste disparu est la représentation d'un modèle censé disparu dont le portrait puis le fantôme se manifesteront aux yeux du locataire du mezzanino du palais Altinengo, Tiberio Prentinaglia, quant à lui, est un être en permanente représentation tout droit issu de la Venise du settecento: "[...] un vrai Vénitien du temps de la Sérénissime République, du temps de Gozzi et de Casanova. Vêtu d'amples vêtements, couvert d'une houppelande, coiffé d'un large feutre» (30). Vivante alliance de contraires: «[...] personnage de comédie et aussi de conte fantastique, il semble fait de plusieurs êtres superposés » (31), cela non seulement dans son apparaitre changeant qui en fait une sorte de Protée baroque, mais aussi dans son rapport au temps, puisqu'il est donné comme le seul, peut-être, à connaître aussi parfaitement la Venise moderne que la 
Venise ancienne, mais encore dans son rapport au réel, puisqu'il est à la fois superstitieux, réaliste et mystificateur à ses heures, autant qu'indispensable sur le plan pratique ; si bien qu'une fois averti de la sorte de logis que recherche son interlocuteur, il lui affirme, en le raccompagnant vers son hôtel, qu'il sait ce qu'il lui faut, une lettre devant l'en informer le lendemain, lui-même devant s'absenter pour un voyage avec Lord Sperling. C'est que pour ce personnage hors du commun, tout étranger résidant à Venise a vocation à devenir un acteur obligé de son théâtre : «Quand on a mis le pied à Venise, on appartient de droit à Prentinaglia, et il n'y a pas à s'en plaindre, car il est d'une ressource infinie » (31).

21 Le soir de leurs retrouvailles, c'est donc la facette " fantastique » de ses multiples rôles qu'il met en scène à l'usage du héros, en alléguant que la disparition du buste témoigne de ce que la cité des Doges se trouve à nouveau le théâtre de phénomènes surnaturels comparables à ceux dont se plaignait d'être assailli Gozzi pour s'être un peu trop moqué des esprits dans certaines de ses comédies féeriques ${ }^{21}$, ou dont jouait parfois Casanova pour mystifier avec succès les uns ou les autres. C'est ce côté mystificateur du personnage que retient surtout le narrateur :

On l'imagine en « tabaro e baüta " paradant, le masque blanc au visage et le tricorne sur sa perruque. Il ne manque pas d'esprit et sa faconde lui en tient lieu à l'occasion. Il s'anime, s'excite, puis tombe dans de longs silences, comme si la ficelle du pantin se fût cassée... À quoi songe-t-il en ces moments d'absorption? À quelque combinaison commerciale? À quelque intrigue amoureuse? Prépare-t-il quelqu'une de ces mystifications auxquelles il se plaît parfois? car c'est encore là un trait de son caractère ; ou médite-t-il une de ces histoires fantastiques qu'il aime à conter et dont il finit par s'effrayer lui-même? car il est, comme je l'ai dit, superstitieux. (33)

Prentinaglia ne reparaîtra pas in vivo sur la scène du récit avant la dernière séquence où, rentrant de voyage, il rend visite à son "ami » blessé, s'accusant théâtralement d'être la cause de son malheur, sans d'ailleurs s'inquiéter des circonstances précises y ayant présidé dans le salon au miroir :

Et, d'une main se frappant la poitrine, de l'autre il désignait le linge de mon pansement. Il était debout, vêtu comme toujours de sa vaste houppelande, avec sa jaune figure de comédie. À son doigt brillait la bague cabalistique dont il avait cacheté la lettre par laquelle il me donnait l'adresse du palais des Fondamenta Foscarini et de la signora Verana. Qu'y avait-il de commun entre cette taciturne Verana au regard sournois et ce Prentinaglia à la figure comme masquée ? Et ce fou de lord Sperling avec sa Casa degli Spiriti ? Prentinaglia était revenu à Rome avec lui et l'avait laissé se rendant à Milan pour un congrès de sciences psychiques. Mais, bientôt, on se retrouverait « sous le Chinois » du Florian! (137)

La bague dont il est question avait été signalée au début de la quatrième séquence du récit, à la réception de la lettre donnant l'adresse du palais Altinengo, renforçant le côté mystificateur de l'informateur, tout en le rattachant en personne aux pratiques occultes en vogue au settecento vénitien: "La bague dont il portait l'empreinte était formée d'une cornaline gravée de signes cabalistiques. Elle avait appartenu à quelque adepte des sciences occultes, comme il y en eut tant à Venise au XVIII ${ }^{\mathrm{e}}$ siècle. Ce bijou s'adaptait parfaitement aux allures de sorcier qu'aimait à se donner le signore Prentinaglia et qui étaient une des facettes de son multiple personnage » (44). Que pour accéder à l'adresse promise, le narrateur doive rompre (c'est le verbe qu'il emploie) « la cire conjuratoire » (44), peut être entendu en un sens ironique à ce moment du récit, mais l'adjectif connote de fait une signification magique: dans cette perspective, le sceau doit être considéré comme porteur d'un pouvoir surnaturel attestant une 
intention - mais laquelle ? À cet égard, cette bague n'est peut-être pas sans lien, dans l'économie analogique du conte, avec d'autres éléments de rupture violente semés tout au long de cette aventure, à commencer par la crise irréparable qui a marqué les trois années précédentes dans l'histoire du héros: «Je savais maintenant ma vie sentimentale brisée et que rien n'en réparerait jamais le désastre » (102). Lors de la rencontre initiale avec Prentinaglia, le narrateur décide de ne pas l'informer de la part déterminante jouée par cette crise dans sa présence à Venise : «Que pouvait-il contre mon mal ? [...] que pourrait-il inventer capable de m'arracher à moi-même? Quel exorcisme sa cabale lui fournirait-elle pour rompre le douloureux sortilège qui me tenait prisonnier?» (35); lors de la promenade sur la lagune dans la journée qui suit, c'est pour «rompre l'angoisse qui [l'] oppressait » à nouveau que le héros avait fait signe au gondolier qui, se méprenant sur son geste, lui avait indiqué la proximité de la Casa degli Spiriti (75) ; lorsqu'au salon jaune, « pendant des heures » écrit le narrateur, le vivant et l'être du miroir se confrontent en silence, « seule, poursuit-il, cette mince feuille de verre s'interposait entre nous, et nous sentions qu'elle ne tarderait pas à se briser, car il fallait qu'elle se brisât » (128); lorsque deux barques se heurtent dans le canal sous le palais dans l'après-midi du jour où se produira l'apparition au miroir ${ }^{22}$, "le choc fut assez rude ", note le narrateur (114); lorsqu'il s'enquiert ensuite, à la clinique, du choc qui lui a valu de s'y retrouver : «- Quel choc ? lui rétorque le docteur, celui de cette lourde porte de miroirs qui s'est détachée de son cadre et qui vous est tombée sur le crâne, en se brisant en éclats! » (133). Une fois seul, il se demandera, en repensant à son compagnon du miroir : « Comme mon propre corps, l'ombre fragile de Vincente Altinengo avait-elle été atteinte par la brusque chute de la porte ? Était-ce par l'accident que notre mystérieux colloque avait été interrompu?» (135) ; c'est enfin au son des «cloches du Redentore et de Santa Eufemia brisa[n]t leurs sons dans l'air cristallin » qu'il salue son image mentale dans l'ultime phrase de la nouvelle.

Le médecin l'avait informé des visites, durant son coma, de la signora Verana, qui l'avait retrouvé au salon baignant dans son sang et avait fait en sorte qu'il fût admis dans la clinique du docteur Bellincioni, ainsi que de celles de Zotarelli, puis de Prentinaglia revenu de voyage « le surlendemain de [son] accident » (134). Mais lors de sa confrontation avec le blessé, Prentinaglia ne se soucie en rien d'en apprendre les circonstances précises, et quitte son "ami» au crâne fêlé en lui lançant "négligemment», après avoir envisagé leurs retrouvailles au Florian «sous le Chinois » :

- À ce propos, mon cher, vous vous souvenez de l'histoire du petit buste du Musée civique dont je vous avais conté la disparition? Eh bien ! il a repris sa place dans sa vitrine. Un beau matin, on l'y a retrouvé, toujours souriant sous sa perruque, mais le mystificateur qui l'avait emporté a dû le laisser tomber, car on y a constaté une fêlure assez visible. On le répare en ce moment... Mais je vous fatigue, mon ami ; au revoir et à bientôt. Je reviendrai. (137)

Dans ses conjectures, le narrateur n'avait jamais pensé à cette disparition en termes de mystification - registre réservé au seul Prentinaglia - ; il penchait nettement pour un vol habilement commis pour des raisons indéchiffrables, tout en se demandant pourquoi le Vénitien avait voulu l'entraîner sur une piste fantastique. C'est ce sur quoi il méditait au Musée civique, le lendemain de leur rencontre, devant la vitrine au buste absent, après avoir dû rebrousser chemin depuis le palais Altinengo dont la propriétaire s'était ce jour-là absentée, repoussant ainsi d'une journée sa visite des lieux : 
L'hypothèse d'un vol paraissant sans doute trop simple à mon ami Prentinaglia, il lui en substituait une autre qui plaisait davantage à son imagination. Pourtant, ce vol, en tant que vol, demeurait intéressant, par ce qu'il y avait d'inexplicable en ses mobiles qui dénotaient une volonté bien particulière. Quelque collectionneur acharné avait-il employé ce moyen de s'approprier cet objet curieux ? Quel rapport cela pouvait-il bien avoir avec les événements surnaturels dont Venise, au dire de Prentinaglia, était devenue le théâtre et au sujet desquels je demeurais fort sceptique? (54-55)

Une fois installé au palais, ses conjectures poursuivront leurs cours selon un registre clairement situé comme relevant exclusivement du genre policier, excluant donc celui du surnaturel :

J'imaginais les différentes hypothèses plausibles qui expliquaient comment le « coup » avait pu être fait; j'en échafaudais qui n'eussent pas été indignes de nos meilleurs romans policiers ; mais j'en excluais soigneusement tout fantastique, car, comme je l'ai dit, j'étais peu enclin à admettre ces interventions surnaturelles [...]. Je ne donnais nullement dans ces billevesées et je préférais supputer par quelles ruses un astucieux voleur avait pu s'approprier ce charmant objet d'art, qui m'avait toujours séduit par la qualité de sa facture et par la physionomie si vivante et si originale du personnage qu'il représentait. (92)

\section{Insouci}

Néanmoins, ce questionnement n'aura qu'un temps; en effet, c'est à des supputations sur la personnalité du modèle représenté qu'il s'abandonnera par la suite - supputations qui ramènent dans le récit la problématique de la mémoire, puisque si le narrateur souligne que la qualité de sa mémoire visuelle en matière d'objets d'arts n'est pas spécifiquement attachée au souvenir de ce buste : «Pour dire vrai, il y avait, dans vingt musées, vingt personnages peints ou sculptés aussi familiers à mon souvenir » (93), il rapporte ensuite que cette faculté mute en hypermnésie s'agissant de cet objet-là ; en effet, son image mentale s'avère bientôt atteindre " une telle précision, qu'il était douteux que le buste lui-même eût été poussé par le modeleur à un pareil point de réalité » (94): de sorte qu'il a l'impression de le contempler pour ainsi dire en grandeur nature comme amplifié par une loupe, ce qui ne laisse pas de l'étonner, sans pour autant l'inquiéter. Par la suite, c'est muni de certains éléments du reste du corps de son modèle supposé que le buste va lui apparaitre mentalement, ces reconstitutions - ou restaurations - fragmentaires ${ }^{23}$ de l'original ne s'unifiant cependant jamais complètement: " Tantôt, il se continuait par les bras, se montrait jusqu'à la taille et même au-dessous. Parfois même il reposait sur ses jambes. Ce phénomène demeurait intermittent, mais se produisait assez souvent. J'avais devant moi, non plus seulement un buste, mais le personnage presque entier » (94). L'habitude s'imposant de "ces jeux visuels ", le héros ne s'étonne plus du phénomène, tandis que le narrateur se distingue a posteriori de lui sur ce plan, en remarquant qu'il eût normalement dû s'en inquiéter, comme d'un symptôme manifeste de l'aggravation de son état :

Ils eussent dû cependant me faire réfléchir et m'indiquer que ces phénomènes relevaient d'une sensibilité anormale et d'un état nerveux plutôt défavorable. Si j'avais raisonné ainsi, j'en aurais conclu que l'existence que je menais au palais Altinengo n'était pas celle qui me convenait et que je ferais bien de me conformer plus exactement aux conseils de mes médecins. Il eût été nécessaire, selon leur recommandation, de joindre au repos prescrit un exercice modéré et de ne pas laisser, ainsi que je le faisais, mes heures inactives s'écouler en vagues rêverie. (95) 
Outre les registres policier et fantastique, la grille médicale est en effet une isotopie interprétative conduite avec esprit de suite par le narrateur, parallèlement aux deux autres, depuis le début de ce récit. Il pourrait s'agir d'hallucinations caractérisées, mais le fait est que le héros ne s'en soucie aucunement, fort heureux au contraire de ce qui lui arrive là, soit le bonheur, dans sa solitude, de rencontrer un "compagnon » imaginaire offert par « le hasard» (95-96).

Cette capacité d'abandon au jeu des circonstances, un peu au-delà du raisonnable, est du reste une caractéristique foncière de sa personnalité telle qu'elle est installée dès le commencement du conte. S'il s'agace de l'acte manqué qui préside à son départ pour Venise, un départ dont cette distraction ou cette négligence une fois reconnues, mais trop tard, lui font songer non sans quelque inquiétude que rien ne l'obligeait, dans son état, à précipiter ainsi un voyage qui risquait de le confronter à des fantasmes peut-être mal maîtrisables, il en prend rapidement son parti. Cette souplesse résulte au fond du point où il en est relativement à l'intérêt de la vie, soit un détachement qui va, on l'a vu, jusqu'à ne pas se soucier du risque d'effondrement présenté par ce palais ; le héros est toujours assez bien averti de ce qu'une approche raisonnable des événements qu'il suscite ou qui lui surviennent pourrait lui représenter de leur étrangeté, mais il passe toujours outre. La raison centrale en est, relativement à tout ce qui lui advient au palais et dans son salon, qu'il a rencontré là, dès le premier abord encore tout extérieur, ce que le récit nomme itérativement un " attrait » irrésistible, en ce qu'il y expérimente à nouveau du désir ; c'est ainsi qu'il se console très vite - non sans fugitivement s'en étonner un peu - de l'absence de la propriétaire lors de sa première visite :

Cette nouvelle me rassura, car d'ici à demain personne ne louerait cet appartement que, je ne savais trop pourquoi, je considérais déjà comme le mien. Elle me déçut, car j'aurais voulu pénétrer immédiatement dans le palais Altinengo. Cet empressement, d'ailleurs, ne fut pas sans m'étonner quelque peu. Depuis mon mal et mes chagrins, depuis que la vie n'était plus pour moi qu'une suite d'actes sans intérêt, indifféremment répétés, c'était la première fois que j'éprouvais un désir (52).

C'est encore une absence de la signora qui provoquera l'événement majeur projetant le héros dans l'univers de ce qu'on pourrait appeler, en détournant un concept de la physique moderne, les 'attracteurs étranges'. Celle qu'il nomme aussi sa "gouvernante » dont le service s'était jusqu'alors signalé par sa perfection silencieuse, s'est en effet avisée de négliger le renouvellement de la provision de bougies; constatant le fait à l'heure de les allumer, le héros endosse son pardessus afin d'aller en quérir à la plus proche boutique ; cependant, réfléchissant par liaison d'idées, qu'il se pourrait que défaillît également la provision de bûches, il se met en quête de l'endroit du logis où sa logeuse les entrepose, ce qui l'amène à reprendre le chemin du jardin où, sur le point de renoncer à une quête décidément infructueuse, il remarque une porte «entrebâillée ». Pénétrant dans ce "réduit » obscur (98), il y observe sur l'un des panneaux de bois, des moulures formant « deux cadres juxtaposés » : « dans l'un de ces cadres subsistait une peinture qui avait dû être un portrait, mais tellement écaillée qu'il était impossible d'y rien discerner que quelques taches de couleur. De l'autre cadre, la toile qu'il avait contenue, détachée par le haut, pendait lamentablement à l'envers » (99). Une fois relevée, cette toile révèle un portrait déchiré dans le sens de la longueur où se présente une première révélation :

Certes, cette seconde peinture avait également souffert et une grande déchirure la séparait presque en deux, mais le personnage qu'elle représentait semblait devoir être assez distinct. En tout cas, une inscription assez bien conservée allait 
m'apprendre son nom. Approchant mes yeux de la toile, j'y lus en effet ces mots :

Vincente Altinengo, nobile Veneziano MDCCLXII. (99)

Le héros a tôt fait d'identifier là le propriétaire des lieux, la date concordant parfaitement avec le style de la décoration du mezzanino, et en particulier du salon jaune; de sorte qu'il l'envisage spontanément comme un hôte lointain qui aurait préparé pour lui ce fastueux décor : «J'étais donc en présence de mon prédécesseur en ces lieux ornés par lui avec tant de luxe et de goût, et je sentis une vive curiosité de l'aspect qu'avait bien pu avoir, de son vivant, ce gentilhomme vénitien qui avait préparé à ma solitude la mélancolique et mystérieuse retraite où j'étais venu chercher le silence de mon cœur et l'oubli de la vie » (100).

Mais il va rencontrer beaucoup mieux encore, puisqu'en fixant la toile à un clou, il y reconnaît, sans doute possible, le répondant exact du portrait mental du buste dérobé qui lui est familier au point d'en faire son compagnon de hasard, sinon de prédestination :

La ressemblance entre le portrait peint et l'effigie modelée était frappante et un singulier hasard me permettait de les identifier l'un à l'autre. L'Inconnu du Musée était l'Altinengo du vieux cadre. Mais pourquoi était-ce à moi qu'avait été réservée cette curieuse révélation dont l'étonnement me tenait adossé au mur, immobile et les yeux fixés sur ces yeux qui me considéraient du fond du passé, d'un regard à la fois lointain et proche, d'un regard presque vivant. (100-101)

Le dernier mot avait été préparé dès la soirée avec Prentinaglia, dans la présentation orientée que celui-ci avait procurée de la disparition du buste :

Vous vous rappelez, n'est-ce pas, dans la salle IV du Musée, celle qui contient le tapis persan offert à la République de Venise par Chah-Abbas, la vitrine où se trouvait un petit buste en pâte tendre? Vous voyez ce que je veux dire, un charmant petit buste de settecento, si expressif, si vivant!

Prentinaglia avait appuyé sur le mot « vivant ». [...]

Prentinaglia me regarda fixement, releva ses gros sourcils et se pencha vers moi :

- Il lui est arrivé, mon cher, qu'il est parti.

- Parti ! Tiberio Prentinaglia fit un signe affirmatif :

- Oui, parti... Depuis une semaine, il a disparu, et toutes les recherches pour le

retrouver ont été infructueuses. (39-40)

Pour peu que le héros, à ce moment de l'aventure, se trouvât encore dans les dispositions qui étaient les siennes lors des premiers temps de son emménagement, quand il échafaudait des hypothèses à la Dupin à propos du buste dérobé, il n'aurait qu'une chose à faire : contacter au plus vite le directeur du Musée civil afin de l'aider à réparer l'absolu déficit d'informations disponible sur l'identification du buste, sinon à retrouver la piste de son voleur. Néanmoins, il n'en fera rien, alléguant l'inutilité d'aggraver la peine supposée du conservateur en lui révélant le nom du modèle - la faiblesse de l'argument en révélant un autre beaucoup plus crédible : les récurrents accès d'angoisse qui lui surviennent à chaque fois qu'il sort du palais, ne serait-ce que pour prendre ses repas, accès dont il convient par surcroît qu'ils n'ont plus rien à voir avec les séquelles de sa crise sentimentale de naguère.

\section{Seuil}

Son tempérament étant de se soumettre, vaille que vaille - hasard et/ou nécessité -, au jeu des circonstances, il décide de se confiner totalement au logis, en admettant par provision que ces regains de nervosité soient occasionnés par le refroidissement lié à l'avancée de l'automne vénitien. Cette sédentarité ne lui pèse en rien, d'autant qu'elle 
lui procure la disparition totale de ses angoisses. Il entre alors dans une existence pour ainsi dire hors du temps que seul le service discret de sa gouvernante rattache encore à la vie ordinaire : il n'ouvre plus un livre, survole distraitement sa correspondance, n'y répond pas, ne reçoit aucune visite. Quant à la compagnie hallucinatoire du corps morcelé du modèle du buste, elle s'est évanouie le jour même de la découverte du portrait de Vincente Altinengo. En revanche, l'attrait qu'il éprouve pour le salon dont il lui doit de jouir hic et nunc, ne cesse d'augmenter, au point qu'il l'a entièrement et précisément mémorisé en tous ses détails :

[...] cette séduction [...] faisait la seule distraction de mon existence sédentaire. Que d'heures passées à examiner avec minutie l'entrelacs des arabesques, les contours des moulures, les dispositions du pavimento ! Je savais exactement les endroits où des fragments de nacre étaient incrustés, parmi les cubes de la mosaïque. Je connaissais tous les jeux de la lumière du jour ou de la clarté des bougies sur les charmants panneaux à figurines dorées. Je savais comment les princesses et les mandarins s'illuminaient selon les heures, leurs miroitements, leurs reflets. J'aurais pu les dessiner de mémoire aussi bien que les sujets du plafond et les rocailles qui, au-dessus de la cheminée, encastraient des petits miroirs en leurs compartiments rococo. (110)

Autrement dit, l'image mentale du salon se superpose à sa réalité, tandis que parallèlement le héros se complaît à en contempler l'image inversée dans le haut miroir en forme de porte factice. C'est alors que le narrateur concède au genre fantastique, quoique du bout des lèvres, que la rencontre avec le portrait de l'ancien propriétaire pourrait éventuellement en relever :

Durant les longues heures de rêvasseries où se consumaient mes journées de solitude, mes regards en revenaient, avec un attrait toujours plus scrutateur, à l'étrange perspective qui s'enfonçait en cet encadrement de marbre et où se reproduisait, avec ma propre personne, tout le décor antique et saugrenu de faïences et de stucs, agencé jadis selon sa fantaisie, en ce vieux palais qui portait encore son nom, par Sa Seigneurie Vincente Altinengo dont un double hasard, que l'on aurait presque pu qualifier de fantastique, m'avait révélé, avec le buste du Musée civique et le portrait du réduit, le visage énigmatique et narquois et le regard vigilant ! [...] (111-112)

La suite va pourtant dévoiler l'illusion de cet apparentement, du moins au sombre sens «cabalistique » où l'entendait Prentinaglia, puisque le héros va se trouver confronté pour son plus grand bonheur à la dimension du merveilleux, de par la manifestation dans ce miroir de l'image, puis progressivement de la matérialisation mobile et autonome, de Vincente Altinengo.

Cette manifestation est précédée d'un effacement de son propre reflet dont il prend le temps de vérifier, durant quelques jours, qu'il ne se produit qu'à la lueur des bougies et n'advient que dans le miroir-porte - une glace à main l'assurant de son reflet aux mêmes heures servant de contre-épreuve.

31 S'il prend note de sa surprise initiale, le narrateur - à la différence de celui du Horla qui confronté au même phénomène et procédant à des vérifications du même ordre, en conçoit la pire terreur -, assure en avoir éprouvé pour sa part une immense joie puisque le voilà soudain devenu, lui l'ordinaire acteur d'une vie sans intérêt, "un personnage exceptionnel » :

[...] cette journée, qui m'avait semblé pareille à toutes les autres, marquait mon entrée dans une existence paradoxale, comme si cette porte de miroirs eût été l'emblème de l'arcade magique par où l'on pénètre dans le monde du mystère et de 
l'inexplicable, au seuil duquel je me trouvais maintenant, sans que rien eût paru

m'y prédestiner jamais (118).

La surnaturalité connotée d'inquiétude et de danger virtuel vers laquelle orientaient le discours de Prentinaglia et ses rémanences dans l'esprit du héros, se révèle merveilleuse à tous les sens du terme, comme le souligne le narrateur: "Jamais je n'eusse songé que pareille aventure pût m'advenir. Mon esprit n'est nullement enclin aux curiosités surnaturelles. J'avais toujours vécu d'une vie qui n'avait rien de merveilleux, au double sens du mot...» (119) - soit, selon Littré, «ce qui, dans un événement, dans un récit, s'éloigne du cours ordinaire des choses » et «ce qui est produit par l'intervention des êtres surnaturels ». Mais s'il s'envisage dès lors comme «transformé en un héros de conte arabe ", ce genre où, comme on sait, la porosité réciproque du surnaturel et du réel est une donnée a priori, il se voit également « devenir à [s]es propres yeux un personnage fantastique » (119).

Il nous faut ici délaisser les raides critères 'structuralistes' de distinction des deux genres - merveilleux vs fantastique -, naguère formulés, non sans simplifications aujourd'hui mieux visibles, par Tzvetan Todorov, et rattacher cette « histoire incertaine » de Régnier à l'univers tout aussi merveilleux expérimenté par le héros de "Spirite - Nouvelle fantastique " qui précède Maupassant et Régnier, au moins pour le $\mathrm{XIX}^{\mathrm{e}}$ siècle français, dans la mise en scène de l'effacement du reflet d'un individu dans un miroir. Une disparition vécue sans aucune terreur par Guy de Malivert, le héros de la nouvelle, puisqu'il a été préparé à en voir émerger une figure féminine dont il a toutes les raisons d'attendre merveilles, pourvu qu'il consente fermement à franchir le seuil de la vie surnaturelle, ainsi que l'en a averti son initiateur et compagnon de cercle $^{24}$. Ce seuil, le héros de L'Entrevue l'avait déjà spontanément passé à son insu, conformément à son tempérament tel que nous avons pu l'observer jusqu'ici :

Cette transformation aurait dû me causer une profonde impression de surprise. Au lieu de m'en émouvoir, je l'acceptai, au contraire, avec une facilité et avec une indifférence qui eussent été explicables si le phénomène en question avait été un phénomène isolé, car j'aurais pu l'attribuer à un trouble visuel momentané. Mais il n'en fut pas ainsi. Le fait se renouvela en des circonstances trop identiques pour qu'il me fût possible d'en conclure à une illusion occasionnelle. (119)

S'occupant dans les trois jours qui suivent à bien vérifier qu'il se trouve échapper, par le fait du miroir-porte, à une "loi physique " qui ne souffre en principe aucune exception, il ne peut donc que souscrire au fait, le seul problème subsistant étant celui de la "mystérieuse intention" (121) qui l'ordonne. En d'autres termes le héros raisonne à l'égard du merveilleux comme il a toujours raisonné dans l'ordre du réel : tout effet constaté comportant sa raison suffisante, il n'est rien d'inexplicable en droit, quoique de l'inexpliqué puisse demeurer en attente. Le lecteur avait d'ailleurs été prévenu, dès le prologue du récit, que le narrateur ne livrerait que des faits :

[...] ce n'est pas une « confession » que j'entreprends ici. [...] Tout ce que je veux me permettre, c'est de noter sur ces feuillets certains faits que j'ose qualifier de singuliers, et qui le paraîtront plus encore par ce qu'il y a d'inattendu à ce qu'ils aient eu pour témoin un personnage de ma sorte, car rien ne me préparait, en effet, au rôle tout involontaire que j'ai joué dans cette histoire. (8-9)

33 En tout état de cause puisque, vérification faite, il n'y a pas lieu de conclure à quelque «illusion occasionnelle» (119) que ce soit relativement à la singularité des faits rapportés, il convient de s'attendre à ce qu'une action intentionnelle y ait prévalu ; et comme Guy de Malivert, c'est bien ce qu'attend le héros: tous deux pressentent 
intuitivement que le vide de leur reflet est en gésine d'apparition. Au quatrième soir en effet, rapporte le narrateur :

Comme les soirs précédents, ayant allumé les bougies, j'étais revenu m'asseoir dans mon fauteuil auprès de la cheminée. J'y demeurai quelque temps, la tête entre mes mains, quand je fus averti, par une sorte de curiosité instinctive, que quelque chose d'intéressant se préparait. Quoi ? je n'aurais pas pu le formuler à moi-même, mais j'en avais l'impression très nette, si nette que mon regard se tourna vers la porte des miroirs, certain que c'était là où se produirait ce que je pressentais d'inattendu. (121)

Mais si, comme pour Malivert, c'est d'abord une vapeur qui émerge dans l'eau du miroir, les deux expériences divergent ici, puisque chez Gautier c'est d'emblée le visage angélique de Spirite qui se manifeste, tandis que l'apparition de L'Entrevue prend d'abord forme de corps vêtu à la mode vénitienne du settencento - un corps dont le visage est voilé :

[...] au fond du salon des stucs reflété, une forme se distinguait, forme encore incertaine et comme vaporeuse, mais qui n'était pas la mienne, car elle se déplaçait tandis que je demeurais immobile. Cette forme humaine remplaçait mon image absente; je m'en rendais mieux compte à mesure qu'elle se faisait plus distincte ${ }^{25}$. Peu à peu, elle le devint assez pour que je pusse distinguer le personnage qui m'apparaissait ainsi. Enveloppé d'un long manteau, il portait un tricorne, avec des culottes courtes et une perruque, mais son visage n'était pas visible. Une sorte de brume le couvrait, tandis que le reste du corps se dessinait assez fermement en grisaille. (121-122)

Une autre différence réside en ce que, dans Spirite, le miroir de Malivert - analogue en cela au miroir où Faust contemple Hélène - ne reflète rien de la pièce où se produit cette manifestation, alors que chez Régnier, la porte-miroir persiste à refléter en perspective inverse le salon des stucs où l'on verra bientôt évoluer l'apparition de Vincente Altinengo; lequel ayant retiré son masque de carnaval, c'est le visage du buste dérobé et du portrait du réduit que le héros reconnaît aussitôt parce que « maints indices » l'y avaient préparé, dès la soirée « sous le Chinois », pense-t-il (122).

- " J'ai tellement envie de savoir si l'on y fait du feu en hiver ${ }^{26}$ " dit Alice à Kitty, à propos de la cheminée du salon reflétée dans le miroir où elle va bientôt pénétrer. Toutes choses égales d'ailleurs, le héros de L'Entrevue, s'il n'a pas le pouvoir de passer de l'autre côté de la porte-miroir, ressemble beaucoup, par le naturel avec lequel il accueille le merveilleux, à l'héroïne de Lewis Carroll : «[...] sa présence me semblait si simple, si naturelle, écrit le narrateur à propos de Vincente Altinengo, que je ne cherchais pas à en comprendre le sens, l'intention et le mystère " (123). De sorte qu'il ne trouve rien de meilleur à faire en cette circonstance que d'accueillir fort convivialement l'apparition:» Salut, Vincente Altinengo, salut! Soyez le bienvenu en cette demeure qui est la vôtre!», quoiqu'il soit persuadé à ce moment que l'autre ne l'entend pas. Durant les nuits qui suivent, le héros se comporte en somme comme un spectateur au théâtre ou au cinéma: il observe les mouvements de l'hôte du salon inverse, en remarquant parallèlement que cette « illusion " prend progressivement une densité, des coloris - en un mot, de la réalité : ce qui ne l'inquiète en rien, mais l'incite à se demander si la réciproque est vraie, autrement dit s'il passe lui-même pour une apparition aux yeux d'Altinengo. Les indices que livre peu après ce dernier à ce propos sont de l'ordre de la surprise ou du trouble car, à la différence du héros, la vision du personnage qui lui est symétrique dans le reflet inversé de son salon lui procure une inquiétude marquée, qui va progressant jusqu'à « l'agitation » : 
Altinengo m'épiait, tantôt ouvertement, tantôt à la dérobée. Parfois, il se levait brusquement $\mathrm{du}$ fauteuil où il venait de s'asseoir, faisait plusieurs tours de chambre, puis s'arrêtait immobile, l'œil au guet, l'oreille tendue. À plusieurs reprises, je le vis se frotter les yeux, comme quelqu'un qui cherche à dissiper quelque illusion visuelle. (126)

Il ira jusqu'à inviter quelqu'un pour lui faire observer le phénomène, sans succès. Le narrateur n'avait jamais songé à impliquer qui que ce soit dans son aventure. Apparaît donc une dissymétrie flagrante entre les deux personnalités : l'une vit très calmement cette entorse insolite aux lois de la réfraction qui donne à sa vie un éclat qu'il n'eût jamais osé espérer, l'autre s'en inquiète manifestement.

Cependant, cette dissymétrie finit par s'effacer au profit de la curiosité suscitée chez l'un et chez l'autre par le phénomène dont ils sont les jouets. Ici, il faut prendre le temps d'envisager la façon dont ce thème a été développé par Régnier au fil du récit. Dans la séquence- prologue, le narrateur se dit peu concerné par les «curiosités esthétiques » ou par l'attention d'autrui à son égard : il sait qu'il a vocation à passer inaperçu et s'en trouve fort bien. Printinaglia, en revanche, ne supporte pas que quiconque séjournant à Venise échappe à sa curiosité toujours aux aguets ; son côté mystificateur inclut par ailleurs le goût de piquer la curiosité du héros, lors de leur conversation au Florian. Ce à quoi il parvient, malgré sa méfiance, puisque le côté « intrigue policière » de l'affaire du buste disparu suscite chez ce dernier une réflexion soutenue pendant un certain temps, jusqu'à ce que prévale son intérêt pour la personnalité et l'histoire du modèle : «Comment avait-il vécu ? Ces questions, je me les posais déjà lorsque je contemplais dans la vitrine du Musée la figure narquoise, voluptueuse et fine de l'inconnu, mais, depuis quelque temps, je me les répétais avec une persistance plus marquée et une curiosité plus passionnée » (92-93) ; cependant, une fois identifié le modèle du buste avec l'ancien propriétaire du palais, «toute curiosité à son égard » disparaît, on l'a vu. En revanche celle qu'il développe à l'égard $\mathrm{du}$ salon depuis sa première illumination nocturne ne cesse de s'intensifier: «Les flammes du foyer s'ajoutaient à celles des bougies, et je suivais leurs mouvements avec une attention et une curiosité qui ne se lassaient jamais »(116), jusqu'à ce que son attention se concentre plus précisément sur «la grande porte glace » où finit par advenir ce qu'on sait, le narrateur notant au passage que son esprit « n'est nullement enclin aux curiosité surnaturelles » (119), en quoi il diffère de Lord Sperling, pour sa part fort « enclin [aux] curiosités de l'au-delà » (77). Ensuite, c'est « par une sorte de curiosité instinctive»(121) que, comme Guy de Malivert, il guette dans le miroir l'imminence de l'inconnu, laquelle devient rapidement " attentive et déjà familière » à l'égard du "compagnon taciturne » de l'autre côté de la glace (124). Enfin, la chose devient réciproque : "Nos yeux s'attiraient avec une curiosité infinie, nos mains se cherchaient " (130). C'est le moment de rappeler que lors de la première évocation mémorielle du buste au Florian, outre le goût du plaisir manifesté par ce visage, ce qui frappe le héros en sa physionomie, c'est « l'expression d'une insatiable curiosité »: « De quoi avait-il été si curieux, ce seigneur vénitien : des secrets de son cœur ou des secrets de l'État? » (39).

On s'aperçoit ainsi que l'incuriosité foncière du héros a muté en son contraire dès le contact retrouvé avec l'image mémorielle du buste dérobé ; puis qu'une fois cette image identifiée avec celle de Vincente Altinengo, il devient surtout attentif aux jeux de lumière sur la décoration du salon, une attention qui se centre progressivement sur la porte-miroir ; jusqu'à ce qu'une « insatiable curiosité » l'identifie, sur ce plan du moins, 
avec Altinengo car demeure une dissymétrie entre eux dans la façon dont il l'envisage : s'il lui semble naturel d'accueillir "sans étonnement ", une fois encore, leur commune aventure, en souhaitant "violemment», de concert avec lui, du moins le semble-t-il, qu'entre eux la glace se brise, c'est qu'il se considère au bout du compte comme son hôte et non comme son égal : «Et puis, hôte de Vincente Altinengo, n'était-il point de simple politesse de tendre la main à son ombre?» (128)

\section{Attracteurs}

Il se peut qu'en cette saillie, comme d'ailleurs dans l'ambivalence du titre du récit, l'auteur prête au narrateur un peu de cette désinvolture des ironistes qu'il fréquentait comme lecteur, sinon comme rêveur hanté sa vie durant par le XviII siècle, ainsi que l'avait observé Léautaud, lequel notait en particulier une connivence avec les Mémoires du comte de Grammont ${ }^{27}$. Comme l'écrit justement Max Milner :

Le monde au-delà, mais aussi le monde en-deçà de la glace, et la mise en présence

de ces deux images se mettant réciproquement en question produit des effets humoristiques qui, par-delà l'ironie propre à l'optique hoffmannesque, font penser à celle qui découlait, pour les conteurs du XVIII ${ }^{\mathrm{e}}$ siècle, d'une vue relativiste du monde ${ }^{28}$.

On pourrait en rester là, mais le conte passe outre en laissant espérer au héros, individu jusqu'alors fort tranquille, n'étaient de récurrentes crises d'angoisse qu'avait efficacement contenues son résolu confinement, qu'il est concevable de briser le miroir aux apparitions comme on brise la glace entre gens de rencontre.

Dans le conte de Régnier intitulé Récit de la Dame des Sept Miroirs, ce sont seulement les vitres de la salle heptagonale que brisent faunes et satyres, avant de se précipiter pour étreindre aux miroirs l'illusion de celle qui s'y mire ${ }^{29}$. Mais changeons d'éclairage en lisant ce conte symboliste qu'est L'Entrevue à la lumière du romantisme allemand : « Les éléments mythiques [dans les contes] sont des débris qui ressemblent aux petits morceaux d'une pierre précieuse éclatée qui seraient éparpillés sur le sol recouvert d'herbe et de fleurs ${ }^{30} »$. Ce sont les frères Grimm qui l'affirment dans leur préface de 1856 aux Kinder- und Hausmärchen. N’y aurait-il pas, dans ce conte vénitien, l'équivalent, aux yeux de l'auteur dont c'est le préféré ${ }^{31}$, de ces petits morceaux dispersés - autant dire ces fragments? Nous les avons recueillis au début de cette étude comme ces " énigmatiques fragments de nacre qu'une fantaisie inexplicable avait incrustés dans la mosaïque du pavimento » (87); mais il est d'autres éléments fragmentaires dans ce conte: ainsi de ce corps "presque entier» fantasmé par le héros selon des hallucinations toujours parcellaires quoique tout à fait précises: "Jamais il ne s'en formait à ma vue un aspect total, mais, par contre, les parties que j'en distinguais étaient toujours d'une remarquable netteté, tout en demeurant fragmentaires » (95). Lors de sa première apparition, c'est en revanche dans sa complète intégrité que se manifeste l'apparence corporelle de Vincente Altinengo, cette restauration étant cette fois corrélée avec les fragments nacrés du pavage : «C'était lui dont les pieds posaient sur le pavage de la mosaïque aux fragments de nacre incrustés et qui se tenait là, incolore, impondérable, presque encore immatériel» (123): quelque chose d'une nécessité analogique se fait ainsi jour à l'approche de la chute du récit, réunissant deux ordres de faits jusqu'alors développés relativement indépendamment. Enfin, nous l'avions vu, tandis que de l'un et l'autre côté de la porte-miroir s'observent face à face Altinengo et le héros, au plus proche de la glace, tel fragment nacré du pavimento est 
comparé à un disque lunaire écorné brillant sur Venise, occupée à se contempler ellemême : «doublée par le miroir de sa lagune circulaire et des eaux insinuées, par mille canaux, en sa mosaïque architecturale » (129). - La pierre précieuse des frères Grimm, la voici nommée Venise retrouvée, peut-être, en cette auto-contemplation en miroir du conte par lui-même, sa mise en abyme si l'on y tient - mais Mallarmé n'avait-il pas composé un merveilleux sonnet « allégorique de lui-même » où " [...] dans l'oubli fermé par le cadre se fixe/ De scintillations sitôt le septuor » d'une constellation reflétée dans celui de ses rimes?

Mais il est une autre pierre précieuse dans ce conte : c'est la cornaline gravée de signes cabalistiques de la bague qui, au début de l'intrigue, scelle d'une « cire conjuratoire » la missive adressant le héros au palais Altinengo; à la fin du récit, lorsque Tiberio Prentinaglia, au terme d'une visite éclair, s'éclipse de la clinique du docteur Bellincioni, c'est encore «la cornaline aux signes de grimoire » que le héros voit «luire » à son index (138). Cette pierre précieuse est, écrit Littré, « une agate demi-transparente, et ordinairement d'un rouge foncé, dont on fait des cachets et autres bijoux ». Rouge est la banquette du Florian; rouge est la croix incrustée au-dessus de la porte de la clinique du bon docteur; rouge est San Michèle, l'île des Morts " aux murailles pourpres et crénelées, pareille à la forteresse du sommeil » (73), tandis qu’à Murano «bout le verre aux fournaises " (73); rouge encore "l'ornement peint à la proue " des barques se heurtant dans le canal, le jour de l'éclipse du reflet du héros dans le miroir (114). Telles sont les concordances des êtres et des choses en ce tissu de la fiction dont Régnier dira ailleurs qu'elle s'aide « du rêve inconscient des choses ${ }^{32}$ ».

Ce rêve, l'œil mental s'en avise plus sûrement que la conscience. Et pas moins l'oreille intime: Cornaline... à quoi cela rime-t-il ? - C'est en tricorne sur la perruque avec masque blanc et baüta que l'on imagine Prentinaglia paradant au carnaval ou sur la scène comique (33); c'est encore en tricorne et dans le même costume que le héros imagine le modèle du buste volé se promenant sous les arcades des Procuraties (56); et c'est encore ainsi coiffé qu'il lui apparaît au miroir le visage embrumé, avant qu'il ne retire son masque, révélant son identité avec Vincente Altinengo (122); mais aussi, c'est pour l'ombre de Catherine Cornaro, reine de Chypre $^{33}$ que rivalisent deux occultistes amoureux que le héros a bien connus (36) ; et encore, ce sont « quatre cornes d'abondance » qui ornent les coins du pavage dans la pièce qui précède le salon jaune (61) ; enfin, nous l'avons vu, c'est une lune écornée qui illumine Venise à son miroir d'eau, tandis que dans l'eau du miroir deux personnages animés de la même curiosité passionnée attendent que l'un d'eux - ou le hasard - brise " la mince feuille de verre » qui les sépare.

On n'en finirait pas de relever les intersignes ou ce que j'ai nommé les 'attracteurs étranges' disposés dans le conte, à l'adresse du lecteur autant que du héros, des attracteurs poétiquement conduits, depuis cette nacre qui se reforme en crâne au prix d'un accent, ce crâne qui se déforme en corne au prix d'un métagramme, comme tel corps se déforme ou se reforme presque entier au prix d'une partie manquante, comme le héros en est à " presque oublier » dans le salon aux stucs ses raisons de s'y trouver (90), comme il est seulement «presque heureux » de retrouver de nuit le labyrinthe vénitien (23), comme il se dit "presque indifférent » à son arrivée, au spectacle de Venise retrouvée (21). Toujours quelque chose défaille à la complétude rêvée ou possible, toujours un détail trouble le jeu des apparences et des circonstances, comme en cet acte manqué ou cette distraction qui inaugure ce voyage, et que le narrateur 
compare à la rupture d'une "maille» dans «le filet» des habitudes anciennes qu'il espérait retrouver par son entremise (22). Toujours quelque chose manque, sinon quelqu'un: ainsi, que voit Vincente Altinengo depuis l'autre côté du miroir? Nous admettons, pour ainsi dire abstraitement, qu'il voit le héros à une époque où celui-ci n'est pas encore devenu le narrateur de leur rencontre. - Mais que savons-nous de l'apparence du héros ? Strictement rien puisqu'il n'est jamais décrit au physique ; nous savons seulement qu'il se définit lui-même par sa vocation à passer inaperçu. Quant à son nom, comme l'écrit Prentinaglia à propos de la date de leur revoir : « Non so, comme nous disons à la vénitienne » (45).

Ce détachement de soi à soi qui caractérise ce personnage était peut-être la condition sine qua non pour que se déroulât, d'un bout à l'autre, le jeu du hasard avec les circonstances et les êtres, tel que l'auteur des Histoires incertaines aime l'observer : c'est en effet "par une coïncidence qui mérite d'être remarquée " (109) que s'effacent des préoccupations du héros les fantasmes relatifs au corps morcelé virtuel du buste, dès lors qu'il a découvert le portrait qui l'identifie; au reste, à bien y regarder, cette découverte annonçait non seulement cet effacement-là, mais aussi celui de sa propre image :

Sur l'un des panneaux les moulures formaient deux cadres juxtaposés, et dans l'un de ces cadres subsistait une peinture qui avait dû être un portrait, mais tellement écaillée qu'il était impossible d'y rien discerner que quelques taches de couleur. (99)

La juxtaposition du portrait indiscernable et de l'envers du portrait déchiré - - de l'autre cadre, la toile qu'il avait contenue, détachée par le haut, pendait lamentablement à l'envers »-, énonce autant leur gémellité qu'elle confirme la loi de perspective inversée réglant les rapports du salon jaune avec son double au miroir, annonçant donc, peut-être, qu'un seul portrait vaut pour les deux pourvu que l'endroit efface l'envers - pourvu aussi que soit réparée, restaurée, la division qui le déchire. C'est là l'une des « coïncidences secrètes » qui ont préparé la rencontre d'un « Parisien d'aujourd'hui » avec un "Vénitien de jadis» (127). D'où la pertinence proprement poétique (au sens de la rigueur ou de l'exigence de ce jeu) de l'ultime 'antinomie générique' formulée par le narrateur, soit refuser, à propos de la rencontre dans le miroir, une interprétation surnaturaliste, au profit d'une logique des «concordances mystérieuses » comme la nomme ailleurs l'auteur ${ }^{34}$ :

Étrange aventure à laquelle rien ne m'avait préparé, mais que j'accueillais sans

étonnement... [...] Elle n'était le résultat d'aucune conjuration et d'aucune

sorcellerie. Quelques petits faits épars m'y avaient conduit insensiblement.

Pourquoi la refuser, puisqu'elle venait à moi ? (127-128)

- Ce qui paraît cohérent, selon la perspective du narrateur au moins, avec l'intention ferme de s'en tenir aux « faits » annoncée dans son préambule. Et non moins cohérent avec l'imprégnation symboliste de la poétique analogique de l'auteur.

De ce dernier, Remy de Gourmont écrivait assez somptueusement, à l'incipit du portrait le concernant dans Le Livre des masques : "Celui-là vit en un vieux palais d'Italie où des emblèmes et des figures sont écrits sur les murs. Il songe, passant de salle en salle, il descend l'escalier de marbre vers le soir, et s'en va dans les jardins, dallés comme des cours, rêver sa vie parmi les bassins et les vasques ». La vérité du personnage semble plus nuancée; si l'imaginaire prévaut chez lui, ce n'est pas sans supports trouvés dans le réel, particulièrement à Venise où la vie "en son humble réalité quotidienne, a quelque chose d'irréel et d'enchantée ${ }^{35}$ ». Les pages de L'Altana concernant le palais Vendramin aï Carmini livrent accès à l'art de la rêverie tel qu'il le pratiquait, comme un 
jeu avec les possibles: si le buste du Museo Civico y demeure le visage préféré du patricien Vendramin, il apparait aussi «contracté de ruse, d'ambition et d'orgueil, intelligent et dangereux ", d'après un portrait de Tiepolo ${ }^{36}$, ou en spectre galant à qui l'on fait bon accueil pourvu qu'il ne retire son masque, "car les fantômes n'ont pas bonne mine et vous n'auriez pas voulu effrayer les deux charmantes jeunes femmes qui, pour vous plaire, auraient revêtu des costumes de votre temps ${ }^{37} »$. Quant à la fameuse porte de miroirs, une poignée de cuivre permet de l'ouvrir, révélant non les lointains du temps mais un placard où " pendre des oripeaux et des défroques de carnaval ${ }^{38}$ ».

Ce n'est pas sur les murs seulement, ni sur une bague de cornaline que s'inscrivent les chiffres de cette cité lacustre sur les eaux de laquelle « les gondoliers, scribes des eaux, [écrivent] du bout de leurs longues rames, à la surface des canaux et sur la page fluide de la Lagune, les lettres d'un alphabet mystérieux ${ }^{39}$ ». Régnier n'a presque rien écrit à Venise même, trop favorable à l'otium sans doute, pour qu'il ne soit pas vaguement malséant d'y vraiment travailler. En revanche, il y a beaucoup erré, en des sortes de dérives avant la lettre, par ces calli "qui semblent être le chemin des hasards favorables ${ }^{40} »$. Et qu'y cherchait-il, sinon l'imprévu, la surprise - ce qu'au fond rencontrait le héros de L'Entrevue, non pas dans la cité mais dans l'athanor d'un salon doré sublimant un passé merveilleusement reviviscent : celui d'une cité magnétique où " tout concourt à une action dont le sens est indifférent ${ }^{41}$ " pour qui lui préfère le charme des coïncidences, des concordances et des analogies offertes, à qui sait voir, par les jeux du hasard et du destin.

\section{NOTES}

1. H. de Régnier, Contes vénitiens, Paris, Le Livre, 1927, "Préface », Collection électronique de la Médiathèque André Malraux de Lisieux. Disponible sur <https://www.bmlisieux.com/archives/ regnie02.htm>.

2. Ibid.

3. H. de Régnier, L'Altana ou la Vie vénitienne (1899-1924), II, Paris, Mercure de France, 1928, p. 226.

4. C'est ce que Régnier représente ailleurs de la cité lacustre ; ainsi dans Le Passé vivant: "Jean de Franois aimait, lui aussi, la ville aux couleurs dansantes, et il sentait avec intensité la beauté et le charme de Venise ; mais il éprouvait une sorte d'anxiété et d'inquiétude. Le lacis compliqué des canaux et des rues qui se coupent, s'entrecroisent, vous ramènent au même point ou vous égarent perfidement, fait d'elle la ville même de l'incertain et de l'imprévu. », Paris, Mercure de France, 1905 p. 160-161. Ou encore, à propos de la lagune « aussi incertaine et mystérieuse que le mystérieux et compliqué labyrinthe vénitien ", L'Altana I, ouvr. cité, p. 43. Pour une approche de l'enjeu générique sous-jacent à la notion d'» histoires incertaines ", voir B. Vibert, "Les "'histoires incertaines' d'Henri de Régnier : des récits minimaux ? », dans S. Bedrane, F. Revaz et M. Viegnes (dir.), Le récit minimal, Paris, Presses Sorbonne Nouvelle, 2012, p. 175-185.

5. S. Basch, Paris-Venise 1887-1932. La "folie vénitienne " dans le roman français, de Paul Bourget à Maurice Debroka, Paris, Honoré Champion, 2000.

6. Voir G. Michaud, "Le thème du miroir dans le symbolisme français ", CAIEF, $\mathrm{n}^{\circ} 11,1959$, p. 199-216. Et en ce qui concerne L'Entrevue, M. Viegnes : “"L'Entrevue” ou la transparence du 
fantastique ", dans B. Vibert (dir.), Henri de Régnier tel qu'en lui-même enfin?, Paris, Classiques Garnier, 2014, p. 113-125.

7. Voir M. Maurin, Henri de Régnier, le labyrinthe et le double, Montréal, P.U. Montréal, 1972.

8. M. Milner, La Fantasmagorie. Essai sur l'optique fantastique, Paris, PUF, 1982.

9. J. Baltrusaitis, Le Miroir, Essai sur une légende scientifique - Révélations, science-fiction et fallacies, Paris, Elmayan, Le Seuil, 1978.

10. La pagination des citations renvoie à l'édition originale : H. de Régnier, Histoires incertaines, Paris, Mercure de France, 1919. Le PDF en est aisément accessible sur le site « Gallica ».

11. En l'occurrence de forme aristotélicienne. Voir C. Michel, "Petite histoire d'une disgrâce ", Introduction à Le démon de l'analogie. Analogie, pensée et invention d'Aristote au $\mathrm{Xx}^{\mathrm{e}}$ siècle, Paris, Classiques Garnier, 2016, p. 7-40.

12. La formule « porte-miroir » n'apparaît pas dans le texte. Les formulations du narrateur à son propos sont de trois sortes. Soit cette fausse porte est faite d'un " grand » ou d'un « haut » miroir (4 occurrences), soit elle est présentée comme une " porte en/de glace » (2 occurrences), soit elle est formée d'une pluralité de miroirs : « porte des miroirs ", « porte de miroirs » (3 occurrences). Cette dernière formulation est autant le fait du narrateur ( 2 occurrences) que celui du docteur qui lui révèle, à la fin du récit, alors qu'il émerge du coma, que » cette lourde porte de miroirs [...] s'est détachée de son cadre et [...] vous est tombée sur le crâne, en se brisant en éclats ». C'est également cette formule 'plurielle' qu'utilise Régnier dans la préface des Contes vénitiens: "singulière porte en miroirs". Comme rien dans le texte du conte ne déploie le scintillement virtuel d'une ornementation de miroirs biseautés, comme c'est le cas dans Spirite de T. Gautier, j'ai préféré neutraliser ce différentiel pour l'analyse, sachant cependant que la formulation «plurielle » peut être rattachée à la pluralité des fragments de nacre ornant le pavage du salon, ainsi qu'aux « éclats » de la porte brisée autour du corps ensanglanté du héros.

13. C'est à propos du feu dans la cheminée de la chambre en miroir : «Comme cela, j'aurai aussi chaud ici que dans la vieille chambre, pensa Alice ». Cité dans P. Mabille, Le Miroir du merveilleux (1940), Paris, Éditions de Minuit, 1962, p. 25. La première traduction française de Through the Looking-Glass, and What Alice Found There (1871) date de 1930. S'il ne semble pas que Régnier se soit intéressé à Lewis Carroll, certaines œuvres entretiennent entre elles, indépendamment de la main qui les conçut, des conversations occultes.

14. «Il ne suffit pas de dire aux songes, aux amours : "Renaissez !" pour qu'ils renaissent; on ne se peut ouvrir la région des ombres qu'avec le rameau d'or, et il faut une jeune main pour le cueillir. » Mémoires d'Outre-tombe, éd. Berchet, Paris, Bordas, «La Pochothèque », 2003-2004, t. I, p. 613.

15. Dans Le Livres des masques, Remy de Gourmont l'identifie comme caractéristique de son coloris en ses vers.

16. «Cette Casa degli Spiriti est un palais situé près de S. Alvise, sur cette partie de la lagune qu'on nomme la «lagune morte », où la marée ne se fait presque pas sentir. C'est une grande bâtisse carrée, demeurée longtemps inhabitée parce qu'elle passait pour être hantée »(37). «C'était une sorte de salon, à peu près carré, à deux fenêtres, entre lesquelles une cheminée de marbre jaune se dressait, surmontée d'un miroir à volutes dorées » (62).

17. L'Altana ou la Vie vénitienne (1899-1924), II, ouvr. cité, p. 253.

18. Ibid., p. 44.

19. L'antiquaire Zotarelli, pourtant peu suspect de sympathie à l'égard de ce qui se trame au palais Altinengo, est en train de fabriquer un de ces modèles réduits associant éléments architecturaux et jardin quand le héros vient chez lui se fournir en meubles : «Quand je pénétrai dans la boutique de la Spadaria, Zotarelli déballait un de ces surtouts en verre de Venise, formés d'un ensemble de vases, de statuettes, de balustrades, de colonnes et de portiques, qui transforment la table au centre de laquelle on les pose en un jardin minuscule, fragile et charmant » (68). 
20. "Souvent je l'avais entendu relater les surprenants résultats atteints par le savant anglais. Phénomènes étranges: lévitation, transport d'objets, apport de fleurs mystérieuses, matérialisations qu'enregistrait la plaque photographique. Après en avoir étudié la théorie, Sperling avait dû vouloir passer à la pratique. De là, l'achat de la Casa degli Spiriti, dont le surnom populaire lui paraissait sans doute de bon augure. Tous ces «miracles » que Crookes obtenait par des médiums appropriés, Sperling les devait chercher par les mêmes moyens » (77-78)

21. Voir les chap. XV-XVII de ses Mémoires.

22. Il s'agit du seul jour dont le narrateur livre «la date exacte», soit le 27 novembre afin de marquer, par le détail circonstancié de ce qui se produit ce jour-là, l'exactitude du souvenir que sa mémoire en a conservé.

23. Outre la rime narrative que cette fragmentation aléatoire offre au jeu des fragments de nacre du pavage plus haut examiné en ce qu'il reflète de plus haut ou de plus vaste, ce morcellement hallucinatoire répond, dans un autre récit de Régnier, au trouble mortel qui s'empare de l'héroïne dans la salle aux miroirs : « Son image s'y multipliait à l'infini. Hertulie autour de soi se vit jusqu'au fond d'un songe où elle perdait le sentiment d'avoir produit tant de fantômes identiques à sa pâleur ; elle s'y sentait dispersée à jamais et, à force de se voir ainsi, ailleurs tout autour d'elle, elle s'y morcela au point que, dissoute en ses propres reflets, exorcisée d'elle-même par cette surprenante magie où elle s'imaginait indéfiniment impersonnelle, ses genoux fléchirent et elle s'affaissa doucement sur le parquet, inanimée, tandis que, dans la chambre solitaire, au-dessus des yeux clos de sa face pâle, les miroirs, en leurs cadres d'or, d'écaille et d'ébène, continuèrent à échanger entre eux l'illusoire aspect de leurs réciproques vacuités. » Hertulie ou les Messages, dans B. Vibert, M. Béghin et A. Kalntzis, Contes symbolistes II, Grenoble, Ellug, 2011, p. 374-375.

24. T. Gautier, Spirite, nouvelle fantastique, IV, Paris, Charpentier, 1886, p. 57.

25. Spirite : «Le miroir, sur lequel ordinairement il ne jetait jamais les yeux, exerçait sur lui une sorte de fascination et absorbait invinciblement son regard. Mais avec quelque fixité qu'il attachât sa vue sur ce point, il ne distinguait rien que ce noir dont les baguettes de cristal faisaient encore ressortir l'intensité mystérieuse. Enfin il crut démêler dans cette ombre comme une vague blancheur laiteuse, comme une sorte de lueur lointaine et tremblotante qui semblait se rapprocher. Il se retourna pour voir quel objet dans la chambre pouvait projeter ce reflet; il ne vit rien. [...] La tache lumineuse du miroir commençait à se dessiner d'une façon plus distincte et à se teindre de couleurs légères, immatérielles pour ainsi dire, et qui auraient fait paraître terreux les tons de la plus fraîche palette. C'était plutôt l'idée d'une couleur que la couleur ellemême, une vapeur traversée de lumière et si délicatement nuancée que tous les mots humains ne sauraient la rendre. Guy regardait toujours, en proie à l'émotion la plus anxieusement nerveuse. L'image se condensait de plus en plus sans atteindre pourtant la précision grossière de la réalité, et Guy de Malivert put enfin voir, délimitée par la bordure de la glace comme un portrait par son cadre, une tête de jeune femme, ou plutôt de jeune fille, d'une beauté dont la beauté mortelle n'est que l'ombre. » T. Gautier, ouvr. cité, V, p. 64-65.

26. L. Carroll, De l'autre côté du miroir/La chasse au Snark, texte français par Henri Parisot, Paris, Flammarion, 1969, p. 19.

27. P. Léautaud, Henri de Régnier, Paris, Bibliothèque internationale d'édition, 1904, p. 21.

28. M. Milner, La Fantasmagorie, ouvr. cité, p. 134-135.

29. H. de Régnier, La Dame aux Sept Miroirs, dans Contes symbolistes II, ouvr. cité, p. 500. Régnier, qui donne en épigraphe à « La Gardienne » ce vers d'Hérodiade : «Je m'apparus en toi comme une ombre lointaine» (Tel qu'en songe, Paris, Librairie de l'art indépendant, 1892, p. 55), pouvait également se souvenir de ceux-ci : «Et tout, autour de moi, vit dans l'idolâtrie/ D'un miroir qui reflète en son calme dormant/ Hérodiade au clair regard de diamant. » 
30. Préface de Wilhelm Grimm à l'édition de 1856 des Kinder- und Hausmärchen. Cité dans P. Beck, Chants populaires, Paris, Flammarion, 2007, p. 8.

31. "De ces divers contes, ce dernier est peut-être celui que je préfère ", écrit-il dans la préface des Contes vénitiens, Paris, Le Livre, 1927.

32. H. de Régnier, "Le Bosquet de Psyché ", Figures et caractères VI, Paris, Mercure de France, 1901, p. 285.

33. Venise 1454-Chypre, 1510. Célèbre pour sa beauté. Il en existe un portrait par Le Titien (1542). 34. «Si [un roman ou un conte] présente un sens inattendu au-delà de ce qu'il semble signifier, il faut jouir de ce surcroît à-demi intentionnel sans y exiger trop de suite et en le considérant comme né fortuitement des concordances mystérieuses qu'il y a malgré tout entre les choses. » «Au lecteur », La Canne de Jaspe, dans Contes symbolistes II, ouvr. cité, p. 221.

35. L'Altana I, ouvr. cité, p. 132.

36. Ibid., II, p. 91.

37. Ibid.

38. Ibid., II, p. 78.

39. Ibid., I, p. 264.

40. Ibid., p. 132.

41. Ibid.

\section{RÉSUMÉS}

Dans ce conte, la symbolique de Venise ancienne et actuelle se trouve analogiquement reliée par tout un réseau d'intersignes à l'appartement où vit le héros-narrateur dans un palais décati, engendrant in fine une coïncidence vécue du passé avec le présent. Ce qui aurait pu être traité en régime fantastique, voire psychiatrique ou même policier, se résout ici en mode merveilleux, après que le lecteur eut été contraint d'hésiter sur la grille de lecture qui convenait à l'interprétation des événements. Le jeu énigmatique des circonstances de l'intrigue permet l'observation de ce que l'auteur nomme « les concordances mystérieuses » que l'école symboliste repère entre les phénomènes, par où la mémoire magique du settecento vénitien hante celle d'un anti héros que sa dépression rend perméable aux manigances merveilleuses du hasard et du destin.

In this tale, the symbolic of the past and present Venice is analogically related, by a complex network of signs, to the flat where the hero-narrator lives in a quasi ruined palace: which finally products for him an astonishing dramatic coincidence between past and present. What could have been written in a fantastic mode only, or as a sort of psychiatric whodunnit, finally appears as an experience of the marvellous; but this only after the reader having been obliged to hesitate about the best interpretative framework to apply. The enigmatic game of the plot's circumstances helps the author to observe wat he calls "the mysterious correspondence" that symbolist school discovers between the phénomenons. Thus, the magic memory of the venitian eighteenth Century will haunt the soul of an antihero fortunately open by his illness to all the marvellous tricks arranged by Chance and Destiny. 
INDEX

Mots-clés : L'Entrevue, Henri de Régnier, conte, Venise, Histoire, mémoire, genre littéraire, fantastique, policier, merveilleux, symbolisme, énigme, ambiguïté, anti-héros, poésie

Keywords : L’Entrevue, Henri de Régnier, tale, History, memory, Literary genre, fantasy, detective story, symbolism, supernatural, enigma, ambiguousness, antihero, poetry

\section{AUTEUR}

JEAN-FRANÇOIS PERRIN

Univ. Grenoble Alpes, 38000 Grenoble, France

fvperr@yahoo.fr 